\title{
Pediatric Hodgkin Lymphoma, Version 3.2021
}

Jamie E. Flerlage, MD, MS ${ }^{1, *}$; Susan M. Hiniker, $\mathrm{MD}^{2, *}$; Saro Armenian, DO, MPH ${ }^{3, *}$; Ellen C. Benya, MD Adam J. Bobbey, MD ${ }^{5}$; Vivian Chang, $\mathrm{MD}^{6}$; Stacy Cooper, MD,*; Don W. Coulter, MD ${ }^{8}$; Branko Cuglievan, MD; Bradford S. Hoppe, MD, MPH ${ }^{10, *}$; Leidy Isenalumhe, MD, MS ${ }^{11}$; Kara Kelly, MD ${ }^{12, *}$; Leslie Kersun, MD, MSCE, MSEd ${ }^{13}$; Adam J. Lamble, MD ${ }^{14}$; Nicole A. Larrier, MD, MSc ${ }^{15, *}$; Jeffrey Magee, MD, PhD ${ }^{16}$; Kwadwo Oduro, MD, PhD ${ }^{17, *}$; Martha Pacheco, MD ${ }^{18, *}$; Anita P. Price, MD ${ }^{19}$; Kenneth B. Roberts, MD ${ }^{20}$; Christine M. Smith, MD ${ }^{21, *}$; Aliyah R. Sohani, MD ${ }^{22, *}$; Erin M. Trovillion, $\mathrm{MD}^{23}$; Emily Walling, MD, MPHS ${ }^{24, *}$; Ana C. Xavier, MD ${ }^{25}$; Jennifer L. Burns ${ }^{26}$; and Mallory Campbell, $\mathrm{PhD}^{26}$

\section{ABSTRACT}

Hodgkin lymphoma $(\mathrm{HL})$ is a highly curable form of cancer, and current treatment regimens are focused on improving treatment efficacy while decreasing the risk of late effects of treatment. The NCCN Clinical Practice Guidelines in Oncology (NCCN Guidelines) for pediatric $\mathrm{HL}$ provide recommendations on the workup, diagnostic evaluation, and treatment of classic $\mathrm{HL}$, including principles of pathology, imaging, staging, systemic therapy, and radiation therapy. This portion of the NCCN Guidelines focuses on the management of pediatric classic $\mathrm{HL}$ in the upfront and relapsed/refractory settings.

J Natl Compr Canc Netw 2021;19(6):733-754 doi: 10.6004/jnccn.2021.0027

${ }^{1}$ St. Jude Children's Research Hospital/The University of Tennessee Health Science Center; ${ }^{2}$ Stanford Cancer Institute; ${ }^{3}$ City of Hope National Medical Center; ${ }^{4}$ Ann \& Robert H. Lurie Children's Hospital of Chicago/Robert H. Lurie Comprehensive Cancer Center of Northwestern University; ${ }^{5}$ The Ohio State University Comprehensive Cancer Center - James Cancer Hospital and Solove Research Institute; ${ }^{6}$ UCLA Jonsson Comprehensive Cancer Center; ${ }^{7}$ The Sidney Kimmel Comprehensive Cancer Center at Johns Hopkins; ${ }^{8}$ Fred \& Pamela Buffett Cancer Center; ${ }^{9}$ The University of Texas MD Anderson Cancer Center; ${ }^{10}$ Mayo Clinic Cancer Center; ${ }^{11}$ Moffitt Cancer Center; ${ }^{12}$ Roswell Park Comprehensive Cancer Center; ${ }^{13}$ Children's Hospital of Philadelphia/ Abramson Cancer Center at the University of Pennsylvania; ${ }^{14}$ Fred Hutchinson Cancer Research Center/Seattle Cancer Care Alliance; ${ }^{15}$ Duke Cancer Institute ${ }^{16}$ Siteman Cancer Center at Barnes-Jewish Hospital and Washington University School of Medicine; ${ }^{17}$ Case Comprehensive Cancer Center/University Hospitals Seidman Cancer Center and Cleveland Clinic Taussig Cancer Institute; ${ }^{18}$ UT Southwestern Simmons Comprehensive Cancer Center; ${ }^{19}$ Memorial Sloan Kettering Cancer Center; ${ }^{20}$ Yale Cancer Center/Smilow Cancer Hospital;

${ }^{21}$ Vanderbilt-Ingram Cancer Center; ${ }^{22}$ Massachusetts General Hospital Cancer Center; ${ }^{23}$ UC San Diego Moores Cancer Center; ${ }^{24}$ University of Michigan Rogel Cancer Center; ${ }^{25} \mathrm{O}$ 'Neal Comprehensive Cancer Center at UAB; and

${ }^{26}$ National Comprehensive Cancer Network.

*Discussion Writing Committee Member.

\section{NCCN CATEGORIES OF EVIDENCE AND CONSENSUS}

Category 1: Based upon high-level evidence, there is uniform NCCN consensus that the intervention is appropriate.

Category 2A: Based upon lower-level evidence, there is uniform NCCN consensus that the intervention is appropriate.

Category 2B: Based upon lower-level evidence, there is NCCN consensus that the intervention is appropriate.

Category 3: Based upon any level of evidence, there is major $\mathrm{NCCN}$ disagreement that the intervention is appropriate.

All recommendations are category $2 \mathrm{~A}$ unless otherwise noted.

Clinical trials: NCCN believes that the best management of any patient with cancer is in a clinical trial. Participation in clinical trials is especially encouraged.

\section{PLEASE NOTE}

The NCCN Clinical Practice Guidelines in Oncology (NCCN Guidelines ${ }^{\circledR}$ ) are a statement of evidence and consensus of the authors regarding their views of currently accepted approaches to treatment. Any clinician seeking to apply or consult the NCCN Guidelines is expected to use independent medical judgment in the context of individual clinical circumstances to determine any patient's care or treatment. The National Comprehensive Cancer Network ${ }^{\circledR}\left(\mathrm{NCCN}^{\circledR}\right)$ makes no representations or warranties of any kind regarding their content, use, or application and disclaims any responsibility for their application or use in any way.

The complete NCCN Guidelines for Pediatric Hodgkin Lymphoma are not printed in this issue of JNCCN but can be accessed online at NCCN.org.

(C) National Comprehensive Cancer Network, Inc. 2021. All rights reserved. The NCCN Guidelines and the illustrations herein may not be reproduced in any form without the express written permission of NCCN.

\section{Disclosures for the NCCN Pediatric Hodgkin Lymphoma Panel}

At the beginning of each NCCN Guidelines Panel meeting, panel members review all potential conflicts of interest. NCCN, in keeping with its commitment to public transparency, publishes these disclosures for panel members, staff, and NCCN itself.

Individual disclosures for the NCCN Pediatric Hodgkin Lymphoma Panel members can be found on page 754. (The most recent version of these guidelines and accompanying disclosures are available at NCCN.org.)

The complete and most recent version of these guidelines is available free of charge at NCCN.org. 
DIAGNOSIS

$$
\begin{aligned}
& \text { Essential: } \\
& \text { - H\&P including: } \\
& \text { - Examination of lymphoid regions, spleen } \\
& \text { - CBC with differential } \\
& \text { - Comprehensive metabolic panel } \\
& \begin{array}{l|}
\text { - Excisional } \\
\text { or incisional } \\
\text { biopsy } \\
\text { - Immunohisto- } \\
\text { chemistry }^{\mathrm{c}} \\
\text { evaluation }^{\mathrm{b}}
\end{array} \\
& \text { pelvis }^{c} \\
& \text { - PET/CT scan }{ }^{d} \text { or PET/MRI scan }{ }^{d} \text { (whole-body) }{ }^{c} \\
& \text { - Pregnancy test for women of childbearing age } \\
& \text { for Supportive Care }{ }^{\dagger} \text { ) } \\
& \text { Useful in selected cases: } \\
& \text { - Fertility preservation }{ }^{\mathrm{e}} \\
& \text { - ECG } \\
& \text { - HIV and hepatitis B/C testing (encouraged) }
\end{aligned}
$$

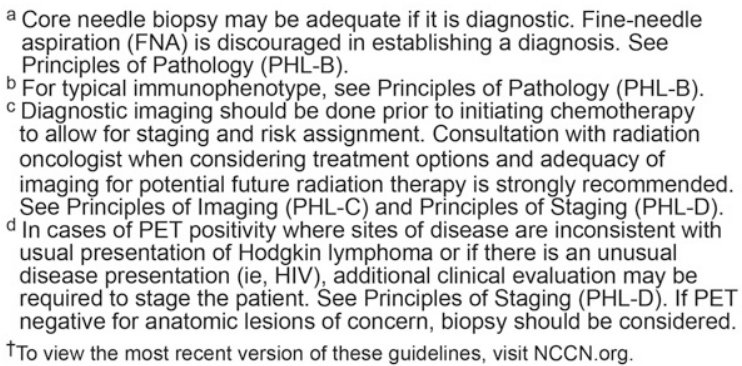$$
\text { - B symptoms (unexplained recurrent fever }>38^{\circ} \mathrm{C} \text { within last month; drenching night }
$$$$
\text { sweats; or weight loss }>10 \% \text { of body weight within } 6 \text { months of diagnosis) }
$$$$
\text { - Erythrocyte sedimentation rate (ESR) and/or C-reactive protein (CRP) }
$$$$
\text { - Echocardiogram (especially if anthracycline-based chemotherapy is indicated) }
$$$$
\text { - Chest } x \text {-ray posteroanterior (PA) and lateral views (if cross-sectional imaging not }
$$$$
\text { available or necessitated to determine bulk of disease for a clinical trial }{ }^{\mathrm{C}}
$$$$
\text { - CT neck/chest/abdomen/pelvis with contrast or CT chest and MRI neck/abdomen/ }
$$$$
\text { - Counseling: Fertility, smoking/drug cessation, psychosocial (See NCCN Guidelines }
$$$$
\text { - PFTs (including diffusing capacity [DLCO] if bleomycin indicated) }{ }^{f}
$$

- Only consider bilateral bone marrow biopsy if there are cytopenias and negative $\mathrm{PET}^{\mathrm{g}}$
CLINICAL PRESENTATION

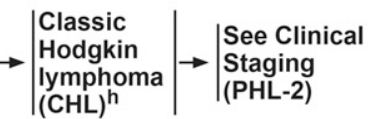

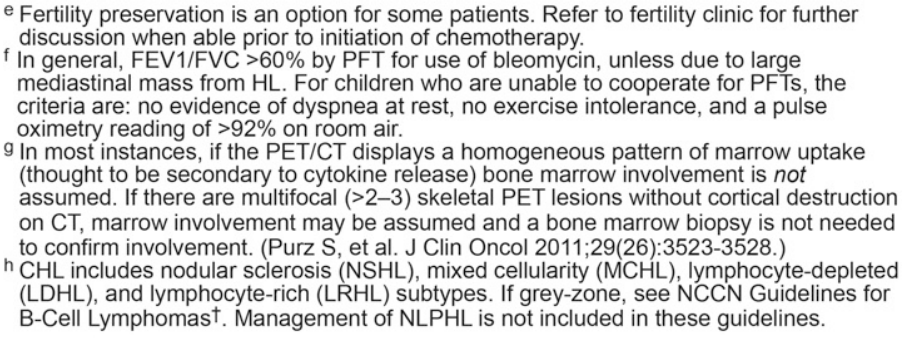

e Fertility preservation is an option for some patients. Refer to fertility clinic for further discussion when able prior to initiation of chemotherapy.

$f$ In general, FEV1/FVC $>60 \%$ by PFT for use of bleomycin, unless due to large mediastinal mass from HL. For children who are unable to cooperate for PFTs, the criteria are: no evidence of dyspnea at rest, no exercise intolerance, and a pulse oximetry reading of $>92 \%$ on room air.

$\mathrm{g}$ In most instances, if the PET/CT displays a homogeneous pattern of marrow uptake (thought to be secondary to cytokine release) bone marrow involvement is not assumed. If there are multifocal $(>2-3)$ skeletal PET lesions without cortical destruction on CT, marrow involvement may be assumed and a bone marrow biopsy is not needed to confirm involvement. (Purz S, et al. J Clin Oncol 2011;29(26):3523-3528.)

h $\mathrm{CHL}$ includes nodular sclerosis (NSHL), mixed cellularity (MCHL), lymphocyte-depleted (LDHL), and lymphocyte-rich (LRHL) subtypes. If grey-zone, see NCCN Guidelines for B-Cell Lymphomas ${ }^{\dagger}$. Management of NLPHL is not included in these guidelines.

\section{Overview}

Classic Hodgkin lymphoma (CHL) is a malignancy involving lymph nodes and the lymphatic system and is generally characterized by the presence of large binucleate or multinucleated neoplastic cells or mononuclear variants, (collectively termed Hodgkin Reed-Sternberg [HRS] cells) in a background of benign inflammatory cells. ${ }^{1}$ Most patients are diagnosed between 15 and 30 years of age, followed by another peak in adults aged 55 years or older. Although the exact etiology is unknown, known risk factors for HL include prior infection with Epstein Barr virus (EBV) and immunocompromising conditions, including immunosuppression after organ transplantation or infection with $\mathrm{HIV}^{2-4}$

The WHO classification divides HL into 2 main types: $\mathrm{CHL}$ and nodular lymphocyte-predominant $\mathrm{HL}{ }^{4} \mathrm{CHL}$ is divided into 4 subtypes: nodular sclerosis CHL; mixed cellularity CHL; lymphocyte-depleted CHL; and lymphocyterich CHL. ${ }^{4} \mathrm{CHL}$ is characterized by the presence of HRS cells in an inflammatory background and expresses CD30, whereas nodular lymphocyte-predominant HL lacks HRS cells but is characterized by the presence of lymphocyte-predominant cells, sometimes termed popcorn cells. It differs from CHL as it is negative for CD30 and positive for CD20. Most cases of childhood HL are CHL; nodular lymphocyte-predominant HL only accounts for $5 \%-10 \%$ of childhood HL. ${ }^{1}$ This rarer subtype is not included in the current Pediatric HL NCCN Guidelines and will be included in future versions.

In 2021, an estimated 8,830 people will be diagnosed with HL in the United States and 960 people will die of the disease. ${ }^{5}$ In adolescents (aged 15-19 years), HL is the most commonly diagnosed cancer ${ }^{6}$ and it is estimated that 4,200 adolescents and young adults (AYAs) aged 15 to 39 years of age were diagnosed with HL in 2020, with 800 of those cases being aged 15 to 19 years. $^{7}$ The incidence is less common in children aged 5 to 14 years; in 2014, a report estimated that 380 children will be diagnosed with HL each year. ${ }^{6}$

The past few decades have seen significant progress in the management of pediatric patients with $\mathrm{HL}$, with estimated 5-year survival rates greater than $98 \%$ after treatment with chemotherapy alone or combined with radiotherapy (RT) ${ }^{8,9}$ However, the potential long-term effects of treatment remain an important consideration. $^{8,9}$ 


\section{CLINICAL STAGING OF CLASSIC HODGKIN LYMPHOMA}

Risk stratification is evolving. This table represents clinical trials with published data. Consider consultation with a center of expertise for patient management; enrollment in a clinical trial is preferred. Clinical trial staging may differ from this table, and close attention to trial eligibility and staging should be followed.

\begin{tabular}{|c|c|c|c|c|}
\hline $\begin{array}{l}\text { Clinical Stage } \\
\left(\text { See ST-1 }{ }^{\star}\right)\end{array}$ & $\begin{array}{c}\text { Bulk } \\
\text { (See PHL-D) }\end{array}$ & $\begin{array}{c}\text { E-lesions }{ }^{j} \\
\text { (See PHL-D) }\end{array}$ & Risk Groupk & \multirow{13}{*}{$\begin{array}{l}\text { See Low-Risk Disease } \\
\text { (PHL-3) } \\
\text { See Intermediate-Risk } \\
\text { Disease (PHL-4) } \\
\text { See High-Risk } \\
\text { Disease (PHL-5) }\end{array}$} \\
\hline \multirow{3}{*}{$\begin{array}{l}\text { IA } \\
\text { IIA }\end{array}$} & No & No & Low risk (per EuroNet-PHL-C1') & \\
\hline & Yes & No & $\begin{array}{l}\text { Low risk (per EuroNet-PHL-C } 1^{\prime} \text { ) } \\
\text { or } \\
\text { Intermediate risk (per AHOD0031) }\end{array}$ & \\
\hline & Yes & Yes & Intermediate risk (per EuroNet-PHL-C1' or AHOD0031) & \\
\hline \multirow[t]{2}{*}{ IB } & Any & No & Low risk (per EuroNet-PHL-C1') & \\
\hline & Any & Any & Intermediate risk (per AHOD0031) & \\
\hline \multirow{4}{*}{ IIB $^{i}$} & No & No & Intermediate risk (per AHOD0031 or EuroNet-PHL-C1') & \\
\hline & No & Yes & $\begin{array}{l}\text { Intermediate risk (per AHOD0031) } \\
\text { or } \\
\text { High risk (per EuroNet-PHL-C1') }\end{array}$ & \\
\hline & Yes & Any & High risk (per AHOD1331') & \\
\hline & Yes & Yes & High risk (per EuroNet-PHL-C1') & \\
\hline \multirow[b]{2}{*}{ IIIA } & Any & No & Intermediate risk (per AHOD0031 or EuroNet-PHL-C1') & \\
\hline & Any & Yes & $\begin{array}{l}\text { Intermediate risk (per AHOD0031) } \\
\text { or } \\
\text { High risk (per EuroNet-PHL-C1') }\end{array}$ & \\
\hline IIIB, IV & Any & Any & High risk (AHOD1331' or EuroNet-PHL-C1') & \\
\hline
\end{tabular}

i Only IIB with bulk was upstaged to high risk in the most recent series of COG clinical trials. The panel acknowledges that current trials have modified these groupings. j E-lesions are defined by the HD10 study as localized involvement of extralymphatic tissue (by contiguous growth from an involved lymph node or in close anatomic relation) that is treatable by irradiation. (Engert A, et al. N Engl J Med 2010;363:640 652; Lister TA, et al. J Clin Oncol 1989;7:1630-1636.)

*Available online, in these guidelines, at NCCN.org. k GPOH-HD-2002: Mauz-Körholz C, et al. J Clin Oncol 2010;28:36803686; EuroNet-PHL-C1: Landman-Parker J, et al. Hematologica/ISHL10, 2016 [Abstract \#P064];101:35; AHOD0031: Friedman DL, et al. J Clin Oncol 2014;32: 3651-3658; AHOD1331: Kelly KM, et al. Br J Haematol 2019;187:39-48; Castellino SM, et al. Klin Padiatr 2020; 232(02):82-83. I Study is complete and data are emerging.
The NCCN Clinical Practice Guidelines Oncology (NCCN Guidelines) for Pediatric HL were developed as a result of meetings convened by a multidisciplinary panel of pediatric HL experts, with the goal of providing recommendations on standard treatment approaches based on current evidence. The NCCN Guidelines currently focus on clinical staging of $\mathrm{CHL}$, and treatment strategies are adapted according to risk. Given the complexity of staging for HL and nuances of treatment regimens and response criteria, the NCCN Pediatric HL Panel recommends a consultation with centers participating in pediatric cooperative group trials. Consistent with NCCN philosophy, participation in clinical trials is always encouraged.

The panel considers the term pediatric to include any patient aged 18 years of age and younger, and the recommendations in the NCCN Guidelines may extend to adolescent and young adult (AYA) patients up to 39 years of age. Across treatment centers, practice patterns vary with regard to AYA patients in terms of whether patients with $\mathrm{HL}$ are treated primarily by pediatric or adult oncologists. These NCCN Guidelines are intended to apply to pediatric patients and may also apply to AYA patients treated in an adult oncology setting.

\section{Diagnosis and Workup for CHL}

For evaluation and initial workup of CHL, the panel recommends that an excisional lymph node biopsy generally be performed (see PHL-1, page 734). Immunostaining for CD30, CD15, CD20, and CD3 is recommended for CHL. Evaluation of an expanded panel of markers (ie, CD45, CD79a, ALK, MUM1, OCT2, and BOB1) should be considered in cases with equivocal or imperfect morphologic features, or to exclude other entities in the differential diagnosis. The HRS cells of CHL express CD30 in all patients, CD15 in most patients, and are usually negative for CD45 and CD3. CD20 may be detectable in a minority of cases. Cases of EBV + CHL may benefit from additional studies such as EBV serology and evaluation for underlying immunodeficiency. For additional information, see "Principles of Pathology" in the algorithm (see PHL-B 1 and 2 of 3, pages 742 and 743).

The workup should include a thorough history and physical examination, including determination of one or more B symptoms (unexplained recurrent fevers $>38^{\circ} \mathrm{C}$ within the last month; drenching night sweats within the last month; weight loss of $>10 \%$ of body weight within 6 months of diagnosis), and examination of lymphoid 
CLINICAL PRESENTATION:

Classic Hodgkin Lymphoma

PRIMARY TREATMENT ${ }^{m, n} \quad$ RESPONSE $^{p}$

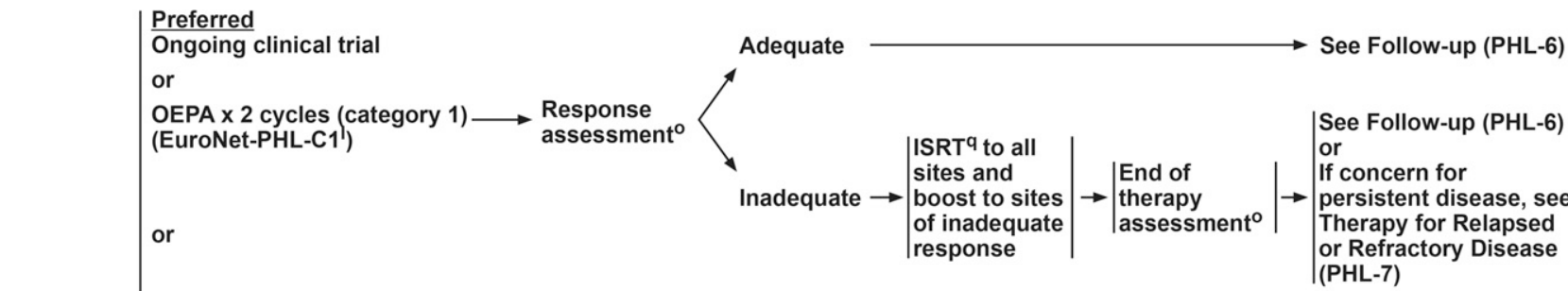

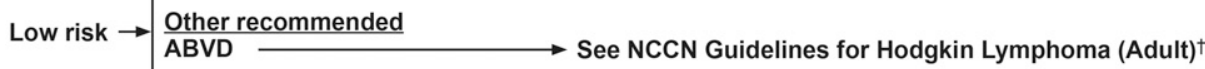

or

Useful in certain circumstances $\quad$ Adequate $\longrightarrow$ See Follow-up (PHL-6)

AVPC $\times 3$ cycles

(for mixed cellularity) $\longrightarrow$ Response

(AHOD0431) assessment $^{\circ}$

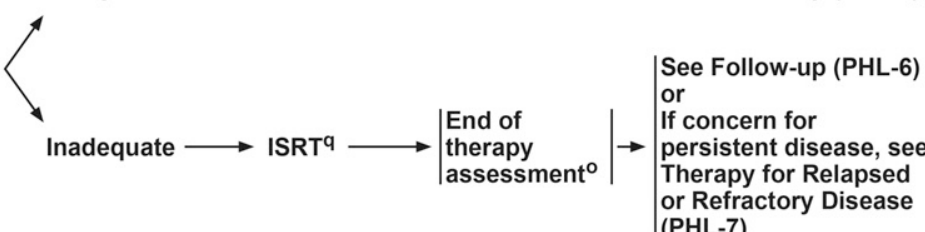

I Study is complete and data are emerging.

$\mathrm{m}$ Regimens are based off of studies with pediatric data.

n See Principles of Systemic Therapy (PHL-E).

- FDG-PET/CT or PET/MRI and contrast-enhanced diagnostic CT or MRI of original sites of disease if not included with PET.

p See Principles of Criteria for Response-Adapted Radiation Therapy (PHL-A)

q See Principles of Radiation Therapy (PHL-F).

†To view the most recent version of these guidelines, visit NCCN.org.

regions and spleen. Other essential components of the workup include standard laboratory tests (complete blood count $[\mathrm{CBC}]$ with differential; erythrocyte sedimentation rate $[\mathrm{ESR}]$ and/or C-reactive protein [CRP]; and a comprehensive metabolic panel). A pregnancy test should be performed before women of childbearing age undergo radiologic testing and/or treatment. HIV and hepatitis B and $\mathrm{C}$ testing is encouraged for patients with risk factors for HIV or unusual disease presentations (see PHL-1, page 734).

PET scans are essential for initial staging and for evaluating residual masses for response during therapy and at the end of treatment ${ }^{10}$ (see "Principles of Imaging," PHL-C 1 of 2, page 744, and "Principles of Staging," PHL-D 1 and 2 of 2, pages 745 and 746 in the algorithm). For staging and risk assessment, diagnostic imaging should be done before initiating chemotherapy or steroids whenever possible including: PET/CT or PET/MRI scans (whole-body); diagnostic contrastenhanced CT of the neck, chest, abdomen, and pelvis; or CT of chest and MRI of the neck, abdomen, and pelvis. PET scans should be assessed by a nuclear diagnostic radiologist experienced in reading Deauville scores for PET-adapted therapy. A posterior-anterior chest X-ray is recommended to determine bulk of disease (mediastinal mass) or as defined by a clinical trial. Consultation with a radiation oncologist is strongly recommended to assist in determining treatment options, assessment of response and application of criteria to determine the need for radiation therapy.

PET interpretation is very challenging given the high sensitivity without specificity. In cases of PET positivity where sites of disease are not consistent with usual presentation of HL or if there are unusual disease presentations (ie, HIV), additional evaluation may be needed to stage the patient. If PET is negative for anatomic lesions of concern, a biopsy may be considered, but is not the standard of care.

In most cases, if the PET/CT displays a homogeneous pattern of marrow uptake, which is thought to be secondary to cytokine release, ${ }^{11,12}$ bone marrow involvement is not assumed. If there are multifocal skeletal PET lesions without cortical destruction on CT, marrow involvement may be assumed and a bone marrow biopsy is not 


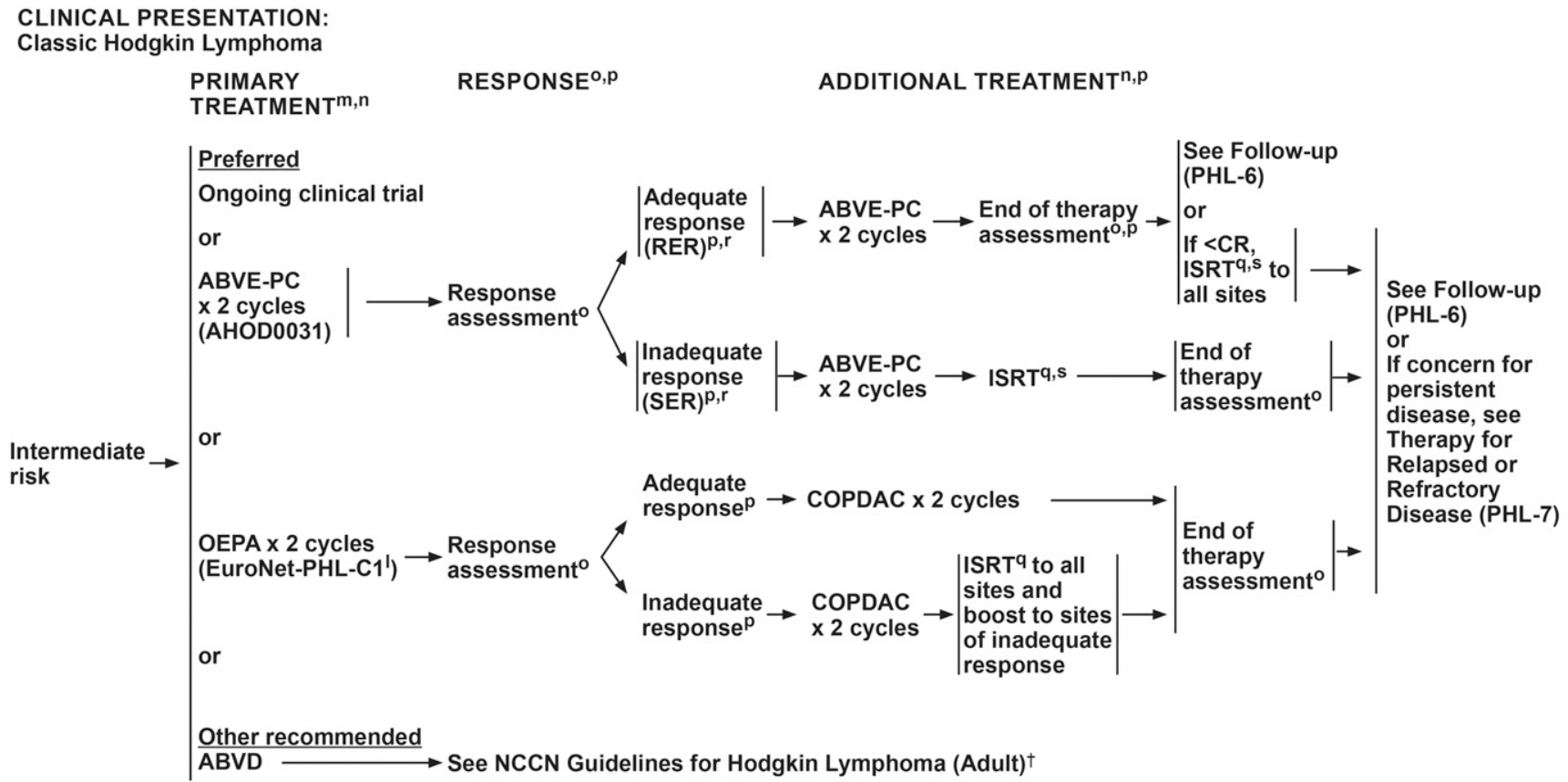

I Study is complete and data are emerging.

$\mathrm{m}$ Regimens are based off of studies with pediatric data.

n See Principles of Systemic Therapy (PHL-E).

- FDG-PET/CT or PET/MRI and contrast-enhanced diagnostic

CT or MRI of original sites of disease if not included with PET.

See Principles of Criteria for Response-Adap

${ }^{r}$ RER = Rapid early responders; SER = Slow early responders
sISRT can safely replace IFRT (see PHL-F).

${ }^{\dagger}$ To view the most recent version of these guidelines, visit NCCN.org.

needed to confirm involvement. ${ }^{13}$ In select cases, if there are cytopenias and the PET scan is negative, a bilateral bone marrow biopsy may be considered.

If anthracycline-based chemotherapy is indicated, an echocardiogram is recommended Pulmonary function tests (PFTs), including diffusing capacity of the lungs for carbon monoxide, are recommended for patients receiving bleomycin-based chemotherapy. In general, an FEV1/FVC of at least $60 \%$ is acceptable for bleomycin use, unless it is lower due to a large mediastinal mass from HL. For children who are unable to cooperate for PFTs, the criteria for bleomycin use are: no evidence of dyspnea at rest, no exercise intolerance, and a pulse oximetry reading of $>92 \%$ on room air.

The panel recommends referral to a fertility specialist for discussion regarding fertility preservation options (eg, semen cryopreservation in male patients, ovarian tissue or oocyte cryopreservation in female patients) before the start of therapy and referrals for counseling as needed that address smoking cessation or substance abuse disorders, and psychosocial concerns. For additional recommendations, see the NCCN Guidelines for Supportive Care (available at NCCN.org).

\section{Clinical Staging and Risk Stratification}

Physical examination and diagnostic imaging evaluations are used to designate the clinical stage. ${ }^{1}$ The most widely used staging scheme for both pediatric and adult $\mathrm{HL}$ is the Ann Arbor Staging, which may include the Cotswolds modification for the prognostic significance of bulky disease ${ }^{1,14,15}$ Staging is generally defined as follows ${ }^{15}$ :

- Stage I: One nodal group or lymphoid organ (eg, spleen, thymus and Waldeyer's Ring)

- Stage II: Two or more nodal groups on the same side of the diaphragm

- Stage III: Nodal groups on both sides of the diaphragm

- Stage IV: Disseminated involvement of one or more extralymphatic organ (eg, lung, bone) with or without any nodal involvement

Additional substaging variables include these terms:

- A: Asymptomatic

- B: Presence of one or more B symptoms

- X: Bulky nodal disease which is nodal mass greater than one-third of intrathoracic diameter 
CLINICAL PRESENTATION:

Classic Hodgkin Lymphoma

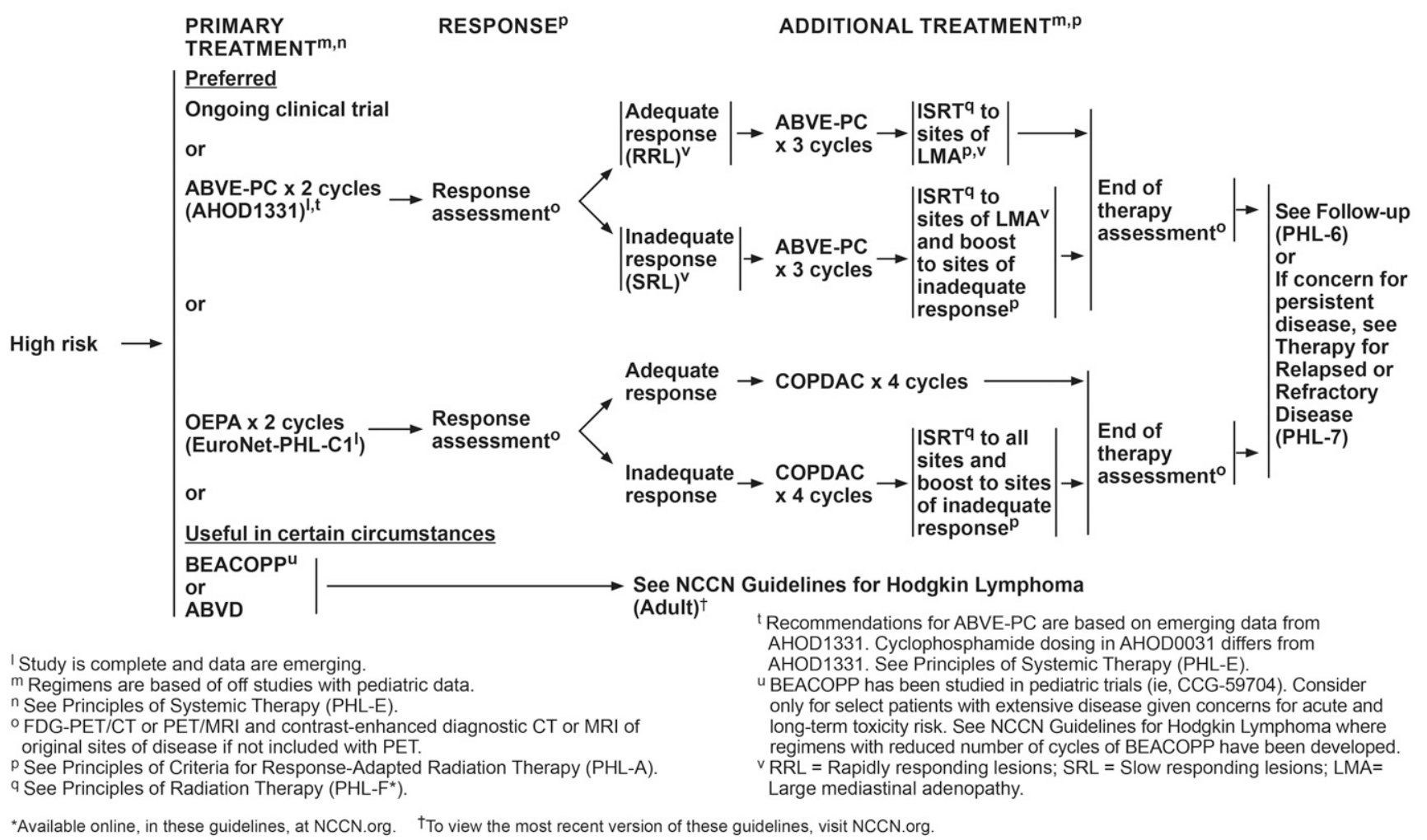

PRIMARY

TREATMENT ${ }^{m, n}$

sites of LMA $^{\mathrm{p}, \mathrm{v}}$

ABVE-PC $\times 2$ cycles $\rightarrow$ Response $(\mathrm{AHOD} 1331)^{1, \mathrm{t}} \longrightarrow$ assessment $^{\circ}$

or

SRT ${ }^{q}$ to inadequat response $^{p}$ If concern for persistent ease, see

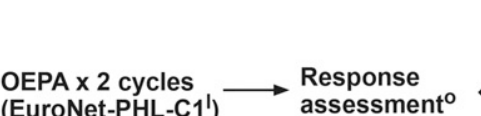
or

Useful in certain circumstances BEACOPPu

See NCCN Guidelines for Hodgkin Lymphoma

Recommendations for ABVE-PC are based on emerging data from 1331. See Principles of Systemic Therapy (PHL-E).

EACOPP has been studied in pediatric trials (ie, CCG-59704). Conside -term toxicity risk. Sich regimens with reduced number of cycles of BEACOPP have been developed.

Large mediastinal adenopathy.

*Available online, in these guidelines, at NCCN.org. †To view the most recent version of these guidelines, visit NCCN.org

on a CXR or as defined by the protocol. Note: pediatric protocols have also defined bulk disease as contiguous extramediastinal nodal mass greater than $6 \mathrm{~cm}$ in the longest transverse diameter or craniocaudal dimension, and EuroNet defines bulk as $>200 \mathrm{~mL}$ (See PHL-D 2 of 2, page 746).

- E: Involvement of extra lymphatic tissue on one side of the diaphragm by limited direct extension from an adjacent nodal site*

*Note: The Ann Arbor staging system is in need of revision as it does not fully represent the current practice in staging pediatric Hodgkin lymphoma. Refer to the original protocol for appropriate staging of "E-lesions." Many protocols today define an E-lesion” as extension from a site of involvement into a surrounding tissue or organ and this does not always indicate stage IV disease. Involvement of an extranodal site that is extralymphatic and does not arise from direct extension is considered to be stage IV disease. The distinction between stage IV disease and E-lesions is not applied uniformly and remains an area in need of international harmonization.
Currently, there is no uniform risk stratification for pediatric HL although several factors are considered to confer a poor prognosis depending on the clinical trial including one or more B symptoms, mediastinal and/or peripheral lymph node bulk, extranodal disease, number of nodal sites, Ann Arbor stage, serum markers for inflammation, gender, and response to initial chemotherapy. ${ }^{1}$ To facilitate the interpretation and comparison of global clinical trials, an international collaborative effort was developed: the Staging Evaluation and Response Criteria Harmonization (SEARCH) for Childhood, Adolescent, and Young Adult Hodgkin Lymphoma (CAYAHL) working group. ${ }^{16}$ As the SEARCH effort for CAYAHL continues, so will the evolution of harmonized risk stratification for pediatric HL. ${ }^{9}$

There are several cooperative groups, including the Children's Oncology Group (COG) (which resulted from a merging of the Pediatric Oncology Group and Children's Cancer Group) and the European Network for Pediatric Hodgkin Lymphoma (EuroNET-PHL). ${ }^{9}$ In the guidelines, the panel has summarized clinical stage and associated risk groups (see "Clinical Staging of Classic Hodgkin Lymphoma,” PHL-2 in the algorithm, page 735) and notes 


\section{FOLLOW-UP AFTER COMPLETION OF TREATMENT} AND MONITORING FOR LATE EFFECTS

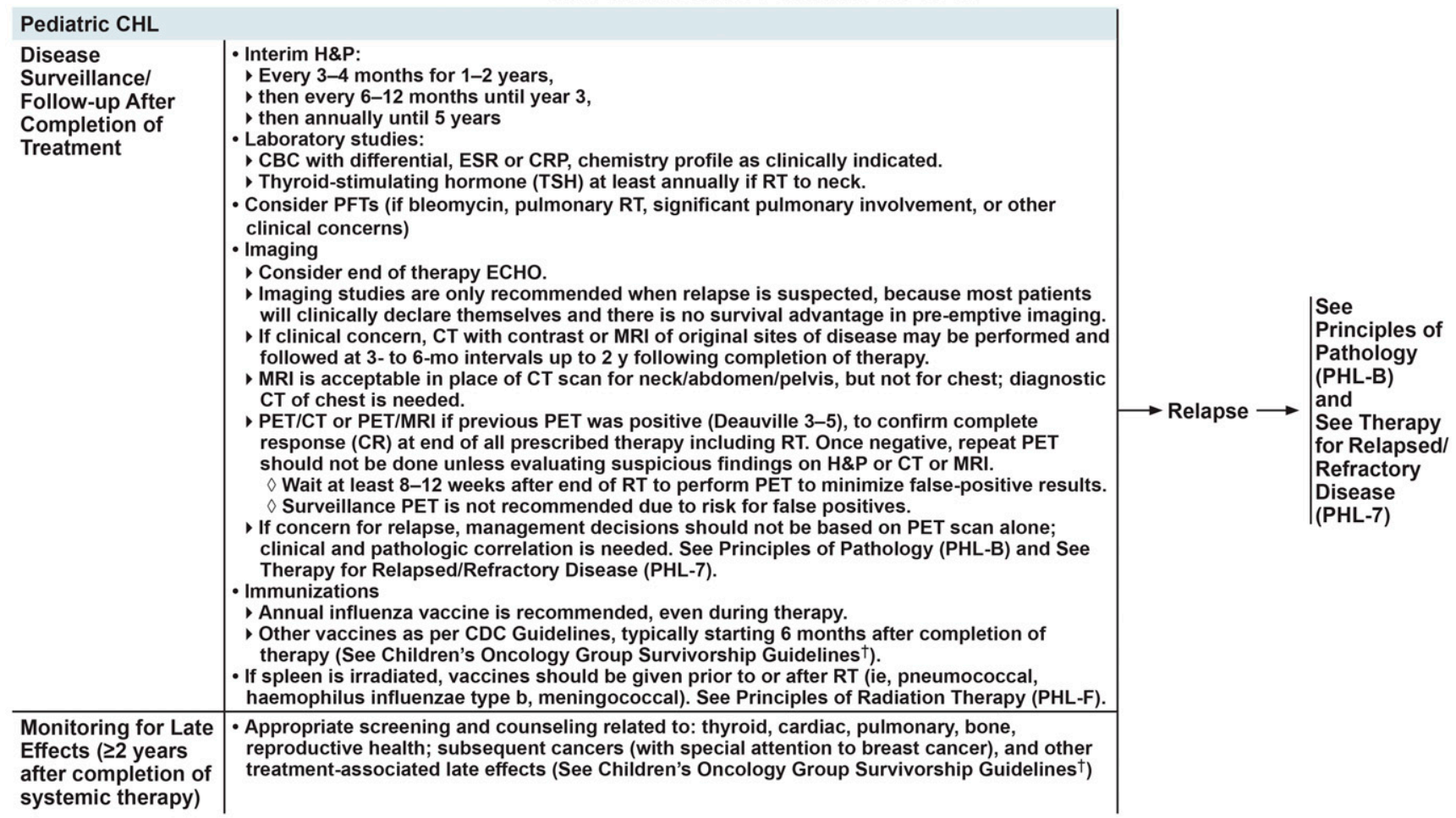

†To view the most recent version of these guidelines, visit NCCN.org.

Version 3.2021, 03/18/21 ๑ National Comprehensive Cancer Network, Inc. 2021. All rights reserved.

The NCCN Guidelines ${ }^{\circ}$ and this illustration may not be reproduced in any form without the express written permission of NCCN.

where the risk groups varied for inclusion onto each trial. Due to the evolving treatment regimens that build on the success of previous trials, enrollment in a clinical trial is always preferred. In addition, given the rarity of these patients and the challenges of staging, the panel recommends considering consultation with a center of expertise for patient management as needed.

\section{Principles of Imaging}

Clinical management of pediatric patients with CHL involves initial treatment with chemotherapy and assessment of treatment response with PET to determine the need for RT. ${ }^{17-19}$

Given the avidity of pediatric lymphomas for $\mathrm{FDG},{ }^{18}$ the Deauville criteria were defined for the interpretation of PET scans based on the visual assessment of FDG uptake in the involved sites (see Deauville Criteria Table in "Principles of Staging," PHL-D 2 of 2, page 746). These criteria use a 5 point-scale (5PS) to determine the FDG uptake in the involved sites relative to that of normal structures such as the mediastinum and the liver. ${ }^{20-22}$ In the 5-PS (Deauville criteria), scores of 1 to 4 refer to initially involved sites. A score of 5 refers to an initially involved site and/or new lesions related to lymphoma, ${ }^{21,22}$ These criteria vary across different protocols because they have yet to be validated in a large pediatric trial. However, interim or end-of-treatment PET scans with a score of 1,2 , or 3 are generally considered "negative" and PET scans with a score of 4 and 5 are considered "positive". ${ }^{23}$ A score of 4 can be difficult to assess when FDG uptake in mediastinal masses cannot clearly be differentiated from thymic uptake or inflammatory reactions, ${ }^{20,24,25}$ and treatment decisions in these cases will require clinical judgment. In addition, Deauville 4 may represent just a single area of persistent disease or failure to respond in any site. The 5PS (Deauville criteria) has been validated in international multicenter trials for PET-guided interim response assessment and risk-adapted-therapy in adult patients with HL.

PET imaging is important as a baseline measurement before therapy to determine the initial sites of involvement and to perform an early response assessment (often after the initial 2 cycles of chemotherapy). ${ }^{10,11}$ The panel recommends diagnostic contrast-enhanced CT or MRI to adequately evaluate all sites of involvement, and PET/CT or PET/MRI for interim and end-of-therapy assessments. In addition, the panel recommends waiting for at least 8 
CLINICAL PRESENTATION:

Classic Hodgkin Lymphoma

\section{SUSPECTED RELAPSED/}

\section{REFRACTORY DISEASE}

\section{RE-INDUCTION THERAPYX}

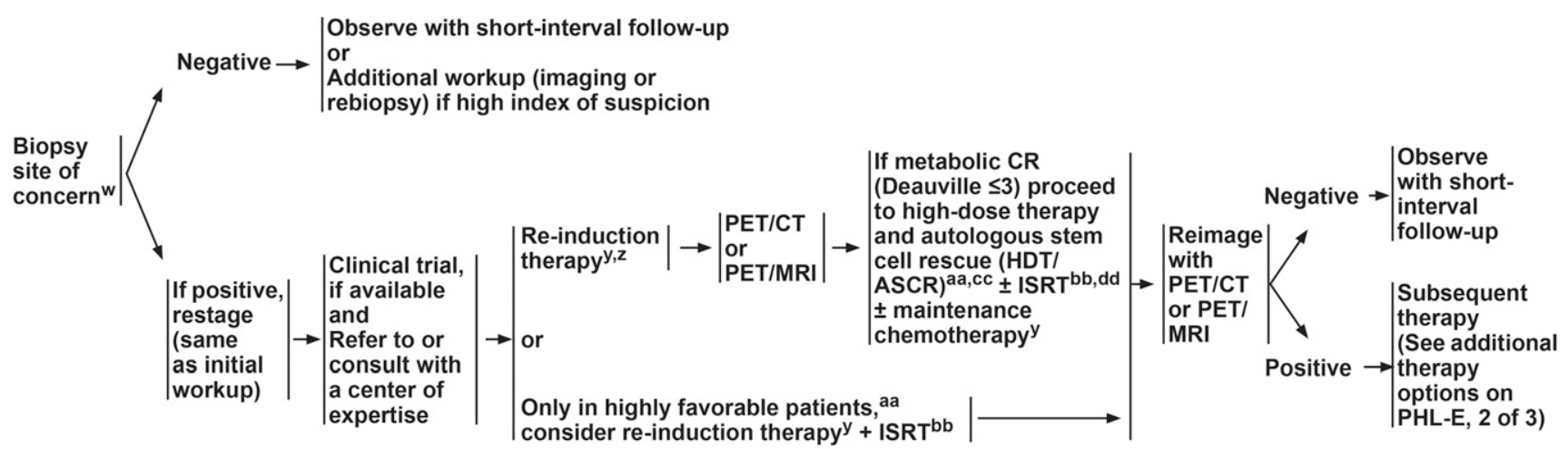

\footnotetext{
w A biopsy must be obtained to confirm relapse and pathology. See Principles of Pathology (PHL-B).

$\times$ There are no data to support a superior outcome with any of the treatment

modalities. Individualized treatment is recommended.

y See Principles of Systemic Therapy for Relapsed or Refractory Disease

(PHL-E, 2 of 3).

z Reasonable to try multiple different re-induction regimens as needed prior to

ASCR to minimize disease burden with a goal of achieving a metabolic CR

prior to transplant. If less than a metabolic CR, proceed to subsequent therapy.
}

aa Recommendations for those who may avoid ASCR: initial stage other
than IIIB or IVB, no prior exposure to RT, duration of CR1 $>1$ year,
absence of extranodal disease or B symptoms at relapse.
bb Strongly consider radiation therapy for selected sites that have not
been previously irradiated.
cc Allotransplant is an option in select patients who relapse post-ASCT
as a category 3 recommendation.
dd RT is usually performed as consolidation after transplant, unless
unable to get to a metabolic CR, then can utilize RT prior to transplant.

PHL-7 to 12 weeks after the end of RT to perform an end of therapy PET scan to minimize false-positive results (see PHL-C 1 of 2, page 744).

In some cases, routine surveillance scans in the first year after completion of therapy may have utility; however, they are recommended to be limited thereafter. During follow-up, scans should only be obtained if there is clinical concern for relapse, or for up to 2 years.

\section{Principles of RT}

RT can be delivered with photons, electrons, or protons, depending on clinical circumstances (see PHL-F 1, 2, and 3 of 4, pages 748-750). Although advanced RT techniques emphasize tightly conformal doses and steep gradients adjacent to normal tissues, the "low-dose bath" to normal structures such as the breasts must be considered in choosing the final RT technique. Therefore, target definition, delineation, and treatment delivery verification require careful monitoring to avoid the risk of tumor geographic miss and subsequent decrease in tumor control. Initial diagnostic imaging with contrast enhanced CT, MRI, PET, ultrasound, and other imaging modalities facilitate target definition.
Data from single institution studies have shown that significant dose reduction to organs at risk (eg, lungs, heart, breasts, kidneys, spinal cord, esophagus, carotid arteries, bone marrow, stomach, muscle, soft tissue and salivary glands) can be achieved with advanced RT planning and delivery techniques such as 4-dimensional CT simulation, intensity-modulated RT/volumetric modulated arc therapy, image-guided-RT, respiratory gating, deep inspiration breath hold, or proton therapy. ${ }^{26-28}$ These techniques offer significant and clinically relevant advantages in specific instances to spare organs at risk and decrease the risk for normal tissue damage and late effects without compromising the primary goal of local tumor control. However, the panel notes that randomized prospective studies to test these concepts are unlikely to be performed since these techniques are designed to decrease late effects, which usually develop $\geq 10$ years after completion of treatment. Therefore, the guidelines recommend that RT delivery techniques that are found to best reduce the doses to the organs at risk in a clinically meaningful manner without compromising target coverage should be considered in these patients, who are likely to enjoy long life expectancies after treatment. 
PRINCIPLES OF CRITERIA FOR RESPONSE-ADAPTED RADIATION THERAPY

\begin{tabular}{|c|c|c|c|}
\hline Regimen $^{a}$ & Risk Group/Stage & Criteria for RT & Protocol Rationale \\
\hline $\begin{array}{l}\text { OEPA/OEPA- } \\
\text { COPDAC } \\
\text { (EuroNet-PHL- } \\
\left(1^{d}\right)^{2,3}\end{array}$ & $\begin{array}{l}\text { Low Risk } \\
\text { - IA/B without E } \\
\text { - IIA without E } \\
\text { Intermediate Risk } \\
\text { - IA/B + E } \\
\text { - IIA + E } \\
\text { - IIB, IIIA } \\
\text { High Risk } \\
\text { - IIB + E } \\
\text { - IIIA+E } \\
\text { - IIIB, IVA/B } \pm E\end{array}$ & $\begin{array}{l}\text { - }<\text { CR on imaging after } 2 \text { cycles of OEPA } \\
\text { CR: Volume reduction }>95 \% \text { and } \leq 2 \mathrm{~mL} \\
\text { CRu: Volume reduction }>75 \% \text { or } \leq 2 \mathrm{~mL} \\
\text { - All IR/HR patients on HD-2002 received RT } \\
\text { - IR/HR patients on } \mathrm{C} 1 \text { (emerging data) received RT only if PET } \\
\text { positive or not in at least PR after } 2 \text { cycles of OEPA, } \\
\text { PR: No CR or CRu and }>50 \% \text { volume reduction or residual } \\
\text { tumor volume }<5 \mathrm{~mL} \\
\text { Note: Volume }=(\mathrm{a} \times \mathrm{b} \times \mathrm{c}) / 2 \text { where } \mathrm{a}, \mathrm{b}, \mathrm{c} \text { are three dimensions } \\
\text { of a node or conglomerate }\end{array}$ & $\begin{array}{l}\text { - 5-year EFS from GPOH-HD-2002 (C1 results } \\
\text { pending) } \\
\text { TG1: } 92 \% \text { (overall) }(92 \%+\mathrm{RT} ; 93 \% \text { without RT) } \\
\text { - TG2/3: } 87 \% \text { 5-year EFS (overall) })^{2} \\
\text { than the } 250 \mathrm{mg} / \mathrm{m}^{2} \text { maximum dose) } \\
\text { - Estimate elimination of RT in } \sim 30 \% \text { of patients } \\
\text { - Refer to EuroNET-PHL-C1 Radiotherapy Manual } \\
\text { No mandatory RT to any sites } \\
\text { RT was only done for poor response, not for all } \\
\text { patients. }\end{array}$ \\
\hline \multirow[t]{2}{*}{ ABVE-PC ${ }^{4,7}$} & $\begin{array}{l}\text { Intermediate Risk } \\
(\mathrm{AHOD} 0031)^{4} \\
\text { - IA, IIA with bulk } \pm \mathrm{E} \\
\text { - IB, IIB without bulk } \pm \mathrm{E} \\
\text { - IIIA } \pm \mathrm{E}^{\mathrm{b}}\end{array}$ & $\begin{array}{l}\text { - Slow early responders (SER) on imaging after } 2 \text { cycles if }<60 \% \\
\text { reduction in product of perpendicular diameters (PPD) }{ }^{\mathrm{c}} \text { for all } \\
\text { target lesions } \\
\text { or } \\
\text { - Rapid early responders (RER) on imaging after } 2 \text { cycles if not in } \\
\text { CR on imaging after } 4 \text { cycles } \\
\text { - CR: } \geq 80 \% \text { reduction in PPD }{ }^{\mathrm{C}} \text { with negative PET at end of } \\
\text { therapy (comparable to Deauville } 1-2 \text { ) } \\
\text { - Consider boost for persistent PET-positive (Deauville } 3-5 \text { ) } \\
\text { lesions at end of chemotherapy. }\end{array}$ & $\begin{array}{l}\text { - 4-year EFS } \\
\text { RER/CR: } 88 \% \text { vs. } 84 \% \text { (+RT) } \\
\text { RER: } 79 \% \text { vs. } 75 \% \text { (+ DECA) } \\
\text { - Response-adapted therapy }\end{array}$ \\
\hline & $\begin{array}{l}\text { High Risk } \\
(\text { AHOD1331 })^{5,6} \\
\text { - IIB with bulk } \\
\text { - IIIB, IV }\end{array}$ & $\begin{array}{l}\text { - Slow responding lesions (SRL) on imaging after } 2 \text { cycles }^{7} \\
\text { - Inadequate or SRL: Deauville } 4-5 \\
\text { - Adequate or rapidly responding lesions (RRL): Deauville } \leq 3 \\
\text { - All large mediastinal adenopathy (LMA) } \\
\text { - Boost for persistent PET-positive lesions (Deauville } 3-5 \text { ) at end } \\
\text { of chemotherapy }\end{array}$ & $\begin{array}{l}\text { - Response-adapted therapy } \\
\text { - Significant decrease in the number of patients } \\
\text { receiving radiation therapy }\end{array}$ \\
\hline $\begin{array}{l}\text { Useful in certain } \\
\text { circumstances: } \\
\text { AVPC } \\
\text { (for mixed } \\
\text { cellularity) } \\
\text { (AHOD0431) }^{1}\end{array}$ & $\begin{array}{l}\text { Low Risk } \\
\text { - IA, IIA without bulk } \\
\text { - For mixed cellularity only }\end{array}$ & $\begin{array}{l}-<\text { CR on imaging after } 3 \text { cycles } \\
\text { - CR: } \geq 80 \% \text { reduction in PPD }{ }^{C} \text { and FDG-PET negative; only } \\
\text { mediastinal nodes }>2 \mathrm{~cm} \\
\text { PET positive (Deauville } 3-5 \text { ): Uptake greater than mediastinal } \\
\text { blood pool }\end{array}$ & $\begin{array}{l}\text { - } 80 \% \text { 4-year EFS } \\
\text { - Those with mixed cellularity histology, who had } \\
\text { a particularly excellent response (4-year EFS } \\
95.2 \% \text { ) } \\
\text { - Limits total doxorubicin dose to } 150 \mathrm{mg} / \mathrm{m}^{2} \text { (less } \\
\text { than the } 250 \mathrm{mg} / \mathrm{m}^{2} \text { maximum dose) }\end{array}$ \\
\hline
\end{tabular}

Involved-site RT (ISRT) is recommended as the appropriate field for HL and can safely replace involved field RT (IFRT) or modified IFRT from earlier trials. ISRT targets the originally involved nodal sites and possible extranodal extension (which generally defines a smaller field than the classic IFRT), ${ }^{29}$ and is intended to spare the adjacent uninvolved organs (such as lungs, bone, muscle, or kidney) when lymphadenopathy regresses after chemotherapy. Treatment planning for ISRT requires the use of CT-based simulation, and additional imaging techniques such as PET and MRI often enhance the treatment planning.

For patients with low or intermediate risk disease, the panel recommends an RT dose of $21 \mathrm{~Gy}$ to all sites of disease. Sites of slow response (usually defined with specific anatomic and/or PET criteria) can receive a boost of up to 9 Gy (total dose, 21-30 Gy). Sites of partial response (PR) should receive a boost of 9-19 Gy (total dose, 30-40 Gy). For patients with high-risk disease, the panel discourages using regimens that require ISRT to all sites of disease. Instead, for bulky disease, a dose of 21 Gy may be considered. The RT doses recommended for sites of slow response and sites of PRs in the low or intermediate risk disease setting are same in this context. The panel notes that residual site RT should only be used when dictated by the protocol or as a boost following standard ISRT.

For patients with relapsed or refractory disease, if no high-dose therapy (HDT) or autologous stem cell rescue (ASCR) is planned, an RT dose of $30 \mathrm{~Gy}$ is recommended. If HDT/ASCR is planned, an RT dose of $30 \mathrm{~Gy}$ to relapsed or refractory sites may be used typically posttransplant, with a consideration of $21 \mathrm{~Gy}$ to initial sites that are no longer present with active disease. If PET positive (Deauville 4-5) after several lines of therapy, RT may be considered to achieve metabolic complete response (CR) before transplant. Boost RT doses of 10-15 Gy (total dose of $40-45$ Gy) to PET positive sites may be considered.

\section{Management of CHL}

In this section, data from select clinical trials that are recommended in the NCCN Guidelines are reviewed to provide a rationale for their inclusion.

\section{Low-Risk CHL}

Approximately $26 \%$ to $34 \%$ of children and adolescents with HL present with low-risk disease. ${ }^{30}$ Outcomes for children and adolescents with low-risk HL are excellent; 


\section{PRINCIPLES OF PATHOLOGY}

Histologic Classification

- Diagnosis should be established according to guidelines in the 2017 WHO Classification of Tumours of Haematopoietic and Lymphoid Tissues. ${ }^{1}$

- There are two types of Hodgkin lymphoma (HL): classic Hodgkin lymphoma (CHL) and nodular lymphocyte-predominant Hodgkin lymphoma (NLPHL). Distinction between these types is important for therapy and prognosis. Management of NLPHL is not included in these guidelines. - There are four morphologic variants of CHL: ${ }^{\mathrm{a}}$

- Nodular sclerosis

- Mixed cellularity

- Lymphocyte-rich

- Lymphocyte-depleted

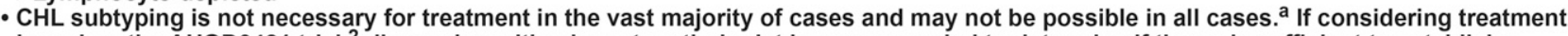
based on the AHOD0431 trial, ${ }^{2}$ discussion with a hematopathologist is recommended to determine if tissue is sufficient to establish a diagnosis of mixed-cellularity subtype.

- $\mathrm{CHL}$ can occur in patients with immunodeficiency (primary immunodeficiency, HIV infection, post-transplant immunodeficiency, and iatrogenic immunodeficiency). Other polymorphic lymphoproliferative disorders and Hodgkin-like lesions are also associated with immunodeficiency and should be distinguished from CHL since management and treatment recommendations differ. These are challenging cases and expert hematopathology evaluation is suggested. Referral to a center of expertise may be necessary.

Tissue Adequacy for Diagnosis

- An excisional or incisional biopsy where possible is recommended. A core biopsy may be appropriate in some settings. ${ }^{\text {b }}$ Fine-needle aspiration (FNA) is discouraged in establishing a diagnosis. ${ }^{c}$

- Ample tissue may be necessary to exclude other entities in the differential diagnosis ${ }^{d}$ and for specific morphologic subtyping. ${ }^{e}$

- Most cases of CHL will not need a bone marrow biopsy; clinically relevant staging information can often be determined from radiologic

findings. See Principles of Imaging (PHL-C).

a The different morphologic variants of $\mathrm{CHL}$ have different clinicopathologic associations and differential diagnoses. Refer to the 2017 WHO Classification for more details. b For example, less accessible anatomic sites such as retroperitoneum.

c Sparse neoplastic cells, extensive fibrosis, and presence of Reed Sternberg-like cells in some conditions other than in $\mathrm{CHL}$ are some reasons a limited biopsy may not be diagnostic.
${ }^{d}$ For example, mediastinal gray zone lymphoma or rare composite tumors of $\mathrm{CHL}$ and primary mediastinal large B-cell lymphoma may not be demonstrable in limited biopsies.

e Fibrotic bands completely surrounding nodules are important in distinguishing nodular sclerosis $\mathrm{CHL}$ from mixed cellularity $\mathrm{CHL}$ but may not be demonstrable in small biopsies. as such, recent trials are focused on modifying treatment (ie, reduction or elimination of specific chemotherapeutic agents or RT) to reduce risk of late effects. ${ }^{30}$ For instance, the German Society of Pediatric Oncology and Hematology Hodgkin's Disease (GPOH-HD) study series has demonstrated that RT can be eliminated from a combined modality treatment scheme for TG-1 patients who experience complete remission after chemotherapy [GPOHHD-95 trial]. ${ }^{31}$

In the GPOH-HD-2002 study, the main goal was to replace a component of chemotherapy (ie, procarbazine with etoposide and dacarbazine) to decrease gonadal toxicity in boys with HL. ${ }^{32}$ In this trial, all patients were aged $<18$ years $(n=573)$; for induction, boys $(n=287)$ received 2 courses of OEPA (vincristine, etoposide, prednisone, and doxorubicin), and girls $(n=286)$ received 2 courses of OPPA (vincristine, procarbazine, prednisone, and doxorubicin). ${ }^{32}$ After chemotherapy, all patients received IFRT to 19 Gy except patients in TG-1 stage who were in CR (residual tumor volume $\leq 95 \%$ and $\leq 2 \mathrm{~mL}$ of the initial volume). In TG-1, the event-free survival (EFS) was $92 \% \pm$ $2.0 \%$, with no significant impact of RT on EFS. ${ }^{32}$ This trial confirmed findings from GPOH-HD-95 that RT could be eliminated in TG-1 patients who experience CR after chemotherapy. $^{31,32}$

Building on the GPOH-HD studies, an international intergroup study for CHL in children and adolescents (EuroNET-PHL C1) aimed to demonstrate a 90\% 5-year EFS in PET-negative patients (TG-1) after 2 cycles of OEPA, and demonstrate that dacarbazine can safely replace procarbazine in consolidation chemotherapy (COPP vs COPDAC) in TG-2 and TG-3 stages without impairing treatment. ${ }^{33}$ In a report from an interim analysis, the 4-year overall survival (OS) and EFS was $98 \%$ and $88 \%$, respectively. ${ }^{33}$ The EFS in TG-1, TG-2 and TG-3 was $87.5 \%, 91 \%$, and $86.6 \%$, respectively $(P=.08)$. The EFS in patients with or without RT was $88 \%$ and $87 \%$. In addition, the EFS was not different between the COPP and COPDAC arms in TG-2 and TG-3. In TG-1, ESR $>30$ or bulky disease was associated with inferior EFS. $^{33}$ This trial suggests that RT can be eliminated in patients who are PET-negative after chemotherapy, and dacarbazine can safely replace procarbazine in COPP, therefore only the COPDAC arm is included in the guidelines. ${ }^{33}$

In the COG AHOD0431 trial, the goal was to evaluate the efficacy of a lower-intensity regimen, AVPC (doxorubicin, vincristine, prednisone, and cyclophosphamide), in 


\section{PRINCIPLES OF PATHOLOGY}

Immunohistochemical Considerations and Ancillary Testing

- Consider clinical differential diagnoses (eg, T lymphoblastic lymphoma) and pathologic differential diagnoses: HL vs. non-Hodgkin

lymphoma (NHL), ${ }^{f}$ CHL vs. NLPHL, HL vs. infection (cytomegalovirus [CMV], Epstein-Barr virus [EBV]), and HL vs. reactive proliferations. ${ }^{g}$

- Diagnosis is based on morphologic AND immunohistochemical findings.

- Typical immunophenotype of HL:

- CHL: Neoplastic cells are PAX5+ (weak), CD30+, CD15+, CD3-, or CD20- (majority). This serves as an essential panel of markers for immunohistochemical evaluation of CHL. Evaluation of an expanded panel of markers (ie, CD45-, CD79a-, ALK-, MUM1+, OCT2-/weak, BOB1-/weak) should be considered in cases with equivocal or imperfect morphologic or immunophenotypic features or to exclude entities in the differential diagnosis.

- NLPHL: Neoplastic cells are PAX5+, CD20+, OCT2+ (strong), CD30-, CD15-, or CD3-. They are also CD45+, CD79a+, BCL6+, EMA+, or MUM1-I weak.

- $E B V+C H L$ cases (EBV often assessed by EBER ISH ${ }^{\mathrm{h}}$ ) may benefit from additional studies, such as EBV serology and evaluation for underlying immunodeficiency.

- Flow cytometry is not helpful in diagnosing HL.' However, it may be helpful in the evaluation of other entities in the clinical or pathologic differential diagnosis.

Pathology Considerations in the Relapse/Refractory Disease Setting

- Pathologic confirmation is necessary to confirm relapse, particularly if $>12$ months after original diagnosis, given the high false-positive rate of PET-CT. Re-biopsy is also recommended for residual PET-avid disease at the end of therapy.

- If original diagnosis slides are available, limited immunohistochemical evaluation may be performed on the relapse/refractory specimen.

- For CHL cases, consider the possibility of misdiagnosis at original presentation, to consider mediastinal gray zone lymphoma lymphoma subtypes. For NLPHL cases, consider the possibility of diffuse large B-cell lymphoma transformation from NLPHL ${ }^{6}$ or reactive lymph node with progressive transformation of germinal centers. ${ }^{7}$

- Prior monoclonal antibody therapy targeting CD30 (for CHL) or CD20 (for NLPHL) may result in weak or negative staining for these antigens by immunohistochemistry.

- There is insufficient data to recommend PDL1 testing by immunohistochemistry as a pre-requisite for checkpoint inhibitor therapy. Robust cut-offs for optimally predicting response to checkpoint inhibitor therapy have not been established.

${ }^{\mathrm{f}} \mathrm{NHL}$ examples, primary mediastinal large B-cell lymphoma, ALK+ anaplastic large cell lymphoma, T-cell/histiocyte-rich large B-cell lymphoma, and EBV+ diffuse large B cell lymphoma.

9 For example, reactive lymph node with $\mathrm{CD} 30+$ immunoblasts (vs. $\mathrm{CHL}$ ) and progressive transformation of germinal centers (vs. NLPHL).

h EBER ISH: Epstein-Barr virus-encoded RNA (EBER) in situ hybridization (ISH).

i Identification of CD4+ CD8dim+ T cells can support a diagnosis of NLPHL, but this population may also be seen in progressive transformation of germinal centers

Neoplastic cells in CHL may also be identified using sophisticated flow cytometry techniques, which are not readily available.

pediatric and AYA patients ( $\leq 21$ years of age) with nonbulky, stage IA and IIA CHL. ${ }^{34}$ All patients $(\mathrm{n}=278)$ were treated with 3 cycles of AVPC, and patients who were not in CR after 3 cycles received 21 Gy of IFRT. Patients who experienced a protocol-defined, low-risk relapse after chemotherapy alone were eligible for an integrated salvage regimen composed of vinorelbine, ifosfamide, dexamethasone, etoposide, cisplatin, and cytarabine, with growth factor support for 2 cycles, and IFRT. ${ }^{34}$ At 4 years, $49.0 \%$ of patients had been treated with 3 cycles of AVPC without RT and $88 \%$ were in CR without receiving HDT/ASCR or $>21$ Gy of IFRT. The OS rate was 99.6\%. Patients with mixed cellularity histology had a 4-year EFS of $95.2 \%$ compared with an EFS of $75.8 \%$ in patients with nodular sclerosis histology $(P=.008) .{ }^{34}$ In this study, a negative PET scan after 1 cycle of chemotherapy (PET1) and an ESR rate $\leq 20$ $\mathrm{mm} /$ hour were associated with a favorable EFS outcome. ${ }^{34}$

\section{NCCN Recommendations for Low-Risk CHL}

For patients with stage IA, IIA, and IB CHL (with or without bulky disease; no E-lesions), the panel recommends enrollment in an ongoing clinical trial or treatment according to EuroNet-PHL-C1 (a category 1 recommendation) as the preferred strategies (see PHL-3, page 736). In certain circumstances, for patients with mixed cellularity histology, 3 cycles of AVPC may be considered per the AHOD0431 trial.

After initial cycles of chemotherapy, patients with adequate response may avoid RT and move to routine follow up. Patients with inadequate response receive ISRT (to all sites and boost to sites of inadequate response per EuroNet-PHL-C1 [see PHL-A 1 of 2, page 741]). Based on an end of therapy PET assessment, patients may either be followed or consider reinduction therapies if there is a concern for persistent disease.

In some pediatric patients with $\mathrm{CHL}$, the $\mathrm{ABVD}$ regimen (doxorubicin, bleomycin, vinblastine, and dacarbazine) may be considered. ${ }^{35-39}$ The panel recommends referring to the NCCN Guidelines for Hodgkin Lymphoma in adults (available at NCCN.org) to review relevant data and context.

\section{Intermediate-Risk CHL}

The phase III COG AHOD0031 study evaluated the role of early chemotherapy response in tailoring subsequent therapy in pediatric intermediate risk HL. ${ }^{40}$ Patients with newly diagnosed intermediate risk $\mathrm{HL}(\mathrm{n}=1,712$; aged 


\section{PRINCIPLES OF IMAGING ${ }^{1-5}$}

Staging or Initial Workup (should be performed within 2-4 weeks prior to initiation of therapy)

- CT neck/chest/abdomen/pelvis with contrast or CT chest and MRI neck/abdomen/pelvis

- Chest x-ray posteroanterior (PA) and lateral views (if cross-sectional imaging not available or necessitated to determine bulk of disease for a

clinical trial)

- PET/CT ${ }^{\mathrm{a}, \mathrm{b}}$ or PET/MRI

- Whole-body is recommended

- Diagnostic-quality CT or MRI is still needed for initial staging

Interim and End-of-Therapy

- PET/CT ${ }^{\mathrm{a}, \mathrm{b}}$ or PET/MRI ${ }^{\mathrm{C}}$

- Wait at least 8-12 weeks after end of RT to perform PET to minimize false-positive results.

- Diagnostic-quality CT with contrast or MRI only for original sites of disease.

Follow-up/Surveillance

- Imaging should only be obtained if significant clinical concern for relapse.

- Example: Follow-up imaging may include diagnostic-quality CT or MRI at 3- to 6-month intervals for up to 2 years.

- PET/CT ${ }^{a, b}$ or PET/MRI is not advised due to risk of false positives.

- May consider repeat PET with persistent positive disease or equivocal finding on post-therapy PET. , $^{\mathrm{b}}$

Relapsed or Refractory (confirmed or highly suspected)

- CT neck/chest/abdomen/pelvis with contrast or CT chest and MR neck/abdomen/pelvis

- PET/CT ${ }^{\mathrm{a}, \mathrm{b}}$ or PET/MRI ${ }^{\mathrm{c}}$

\footnotetext{
a PET should be read by an experienced nuclear diagnostic radiologist experienced in reading Deauville scores for PET-adapted therapy. PET/CT should be obtained in accordance with American College of Radiology (ACR) practice guidelines.

$\mathrm{b}$ In cases of PET positivity where sites of disease are inconsistent with usual presentation of Hodgkin lymphoma or if there is an unusual disease presentation (ie HIV), additional clinical evaluation may be required to stage the patient. See Principles of Staging (PHL-D). If PET negative at anatomic lesion of concern, biopsy should be considered. In most instances, if the PET/CT displays a homogeneous pattern of marrow uptake (thought to be secondary to cytokine release) bone marrow involvement is not assumed. If there are multifocal $(>2-3)$ skeletal PET lesions without cortical destruction on CT, marrow involvement may be assumed. c If PET/MRI obtained, diagnostic CT of chest is needed
}

$<22$ years) received 2 cycles of ABVE-PC (doxorubicin, bleomycin, vincristine, etoposide, cyclophosphamide, and prednisone) followed by early response assessment with PET/CT. For patients who experienced adequate response (rapid early response [RER], based on anatomic criteria), 2 additional cycles of ABVE-PC were given followed by response assessment. RERs with CR $(80 \%$ or greater reduction in the PPD or a return to normal size for all target lesions, plus no residual extramediastinal nodal mass $>2.0 \mathrm{~cm}$, no residual disease in nonmeasurable sites, and a negative gallium or FDG-PET scan) were randomly assigned to IFRT (21 Gy) or observation, and RERs with less than CR were nonrandomly assigned to receive IFRT. In patients who experienced inadequate response (slow early response [SER]) after 2 cycles of ABVE-PC, they were randomly assigned to receive or not receive 2 cycles of chemointensification with DECA (dexamethasone, etoposide, cisplatin, and cytarabine) followed by 2 additional cycles of ABVE-PC. All patients in the SER group received IFRT.

The overall 4-year EFS was $85 \%$ (86.9\% for RERs and $77.4 \%$ for SERs; $P<.001$ ), and the 4 -year OS was $97.8 \%$ (98.5\% for RERs and $95.3 \%$ for SERs; $P<.001) .{ }^{40}$ In RER patients who experienced CR at the end of chemotherapy, there was no significant difference in the 4-year EFS rate between patients who received IFRT versus those who did not receive IFRT ( $87.9 \%$ vs $84.3 \%$, respectively). For SER patients who received either DECA or no DECA, the 4-year EFS was $79.3 \%$ vs $75.2 \%$, respectively $(P=.11)$. PET response imaging was not required but was obtained on the majority of patients as part of clinical care. Analysis of these data demonstrated that SER patients with PET positive lesions after 2 cycles had a marginal improvement in EFS on the DECA arm $(70.7 \%$ vs $54.6 \%, P=.05)$. Overall, this study showed that RT can be omitted in RERs with CR at the end of chemotherapy, and that augmenting chemotherapy in SERs with PET-positive disease may be beneficial. ${ }^{40}$

Other clinical studies evaluating the intermediate risk group (TG-2) include the GPOH-HD-2002 and EuroNETPHL C1 trials ${ }^{32,33}$ as described under "Low-Risk CHL" (page 741).

NCCN Recommendations for Intermediate-Risk CHL For patients with stage IA/IIA CHL (with bulky disease; with or without E-lesions), IB CHL (with or without bulky disease or E-lesions), IIB CHL (no bulky disease; with or without E-lesions), and IIIA CHL, the panel recommends 


\section{PRINCIPLES OF STAGING ${ }^{a}$}

- These are only guiding principles of initial staging adapted from criteria of various protocols. This table is not intended to replace protocol specific staging. Refer to applicable study protocol for complete staging details.

- While these principles are based on panel consensus, this remains an area of ongoing research.

\begin{tabular}{|c|c|c|}
\hline $\begin{array}{l}\text { Site } \\
\text { involvement }\end{array}$ & $\begin{array}{l}\text { Imaging } \\
\text { modality } \mathbf{b}, \mathbf{c}, \mathbf{d}\end{array}$ & \\
\hline $\begin{array}{l}\text { Peripheral } \\
\text { nodes }\end{array}$ & PET/CT or PET/MRI & $\begin{array}{l}\cdot \geq 2 \mathrm{~cm} \text { is considered involved on CT scan } \\
\cdot<2 \mathrm{~cm} \text { if FDG-PET positive }\end{array}$ \\
\hline \multirow{2}{*}{ Splenic } & Ultrasound & $\begin{array}{l}\text { - Any lesion large enough to characterize unless imaging characteristics indicate an alternative etiology irrespective of the } \\
\text { FDG-PET result }\end{array}$ \\
\hline & PET/CT or PET/MRI & $\begin{array}{l}\text { - Focal PET-positive lesions that are confirmed by CT or MRI or ultrasound } \\
\text { - Splenic involvment has to be focal lesions and not diffuse uptake or splenomegaly }\end{array}$ \\
\hline Lung $^{\mathrm{e}}$ & $\mathrm{PET} / \mathrm{CT}$ & $\begin{array}{l}\text { - E-lesions: Extra-lymphatic structures (lung lesions) contiguous with nodal masses are considered to be E-lesions } \\
\text { - At least } 1-2 \text { small foci (between } 5-10 \mathrm{~mm} \text { ) within whole lung if no other etiology is suspected } \\
\text { - At least } 1 \text { intrapulmonary focus }>1 \mathrm{~cm} \text { on CT if no other etiology is suspected } \\
\text { - PET-positive lesions }<1 \mathrm{~cm} \text { if no other etiology is suspected } \\
\text { Note: If all lesions are exclusively in } 1 \text { lung, then only this particular lung is considered as involved. However, even if there } \\
\text { is just one additional smaller focus found within the other lung, then both lungs are considered involved. }\end{array}$ \\
\hline \multirow[b]{2}{*}{ Liver } & Ultrasound & $\begin{array}{l}\text { - Any lesion large enough to characterize unless imaging characteristics indicate an alternative nature irrespective of the } \\
\text { FDG-PET result }\end{array}$ \\
\hline & PET/CT or PET/MRI & $\begin{array}{l}\cdot \geq 1.5 \mathrm{~cm} \text { on CT, if FDG uptake greater than or equal to that of normal liver or spleen parenchyma, respectively, should be } \\
\text { considered positive } \\
\cdot<1.5 \mathrm{~cm} \text { on CT, if FDG uptake greater than that of normal liver or spleen parenchyma should be considered positive }\end{array}$ \\
\hline \multirow[t]{2}{*}{ Bone marrow } & Bilateral biopsy & $\begin{array}{l}\text { - Positive by histopathology on previous high-risk trials; current trial recommendations are based on FDG-PET alone } \\
\text { - European-based GPOH-HD-2002 staging: Not recommended }\end{array}$ \\
\hline & PET/CT or PET/MRI & - $\geq 3$ FDG-PET-positive lesions in bone marrow without cortical bone destruction \\
\hline Bone & PET/CT or PET/MRI & - FDG-PET-positive lesion with cortical bone destruction on CT or MRI ${ }^{f}$ \\
\hline
\end{tabular}

a Clinical interpretation of staging at diagnosis should not be based on

${ }^{\mathrm{c}}$ There may be PET-avid lesions that need clinical correlation to determine if it is related to lymphoma.

reports alone. Treating clinician notes should summarize interpretation of sites of involvement prior to initiation of treatment.

${ }^{b}$ PET should be read by an experienced nuclear diagnostic radiologist experienced in reading Deauville scores for PET-adapted therapy.

d See Principles of Imaging (PHL-C).

e There are inconsistencies in staging between protocols and providers. Careful attention to

staging of lung involvement is important as it may change the risk group of the patient.

$f$ Lewis J et al. Pediatr Blood Cancer 2020;67(4):e28142.

enrollment in an ongoing clinical trial or treatment according to AHOD0031 or EuroNet-PHL-C1 as the preferred strategies (see PHL-4, page 737).

For AHOD0031 regimen, after 2 initial cycles of ABVE-PC, patients with adequate response are treated with 2 additional cycles of ABVE-PC. Based on an end of therapy PET assessment and CR achievement by CT criteria, patients may either be followed, or treated with ISRT to all sites if less than CR. Patients with inadequate response receive 2 additional cycles of ABVE-PC and ISRT (see PHL-A 1 of 2, page 741).

For the regimen based on EuroNet-PHL-C1, after 2 initial cycles of OEPA, patients with adequate response are treated with 2 cycles of COPDAC. Patients with inadequate response are treated with 2 cycles of COPDAC and ISRT (to all sites and boost to sites of inadequate response; see PHL-A 1 of 2, page 741). In both cases, based on an end of therapy PET assessment and CT scan, patients may either be followed or considered for biopsy to confirm persistent active disease.

As recommended for low-risk $\mathrm{CHL}$, the $\mathrm{ABVD}$ regimen may be considered for some pediatric patients. ${ }^{35-39}$ The panel recommends referring to the adult NCCN
Guidelines for Hodgkin Lymphoma to review relevant data and context.

\section{High-Risk CHL}

In a study by the Pediatric Oncology Group (P9425), the efficacy of ABVE-PC in intermediate or high-risk HL $(n=216$; age $<22$ years) was assessed. ${ }^{41}$ After 3 cycles of ABVE-PC, early response was evaluated and patients with RER based on anatomic criteria received IFRT (21 Gy) and those with SER received 2 additional cycles of ABVE-PC (total of 5 cycles) followed by IFRT. Patients were also randomly assigned to receive or not receive dexrazoxane to evaluate its effect as a protectant from anthracycline-induced cardiac and bleomycin-induced pulmonary toxicity. Of 209 evaluable patients, the 5-year EFS was $84 \%$ (84\% and $85 \%$ for intermediate- and high-risk patients, respectively; $P=.87){ }^{41}$ The EFS differed between patients with large mediastinal adenopathy (LMA) versus those without LMA $(80 \% \pm 4 \%$ vs $91 \% \pm 3 \%$; $P=.03$ ). However, use of dexrazoxane did not affect EFS, but may increase risk for acute toxicity, especially typhlitis. The 5-year OS was 95\% and did not differ between RER and SER groups. ${ }^{41}$ Overall, this trial allowed a reduction in alkylator and anthracycline exposure in $63 \%$ of patients. 
PRINCIPLES OF STAGING ${ }^{a}$

ASSESSMENT OF BULK DISEASE

\begin{tabular}{|l|ll|}
\hline Site involvement & US-based protocols & European-based \\
\hline Peripheral nodes & $\begin{array}{l}\text { - Contiguous extramediastinal nodal aggregate }>6 \\
\text { cm in the longest transverse diameter (transaxial } \\
\text { measurement) or craniocaudal dimension } \\
\text { (measured on reformatted CT) }\end{array}$ & • Volume of the largest contiguous lymph node mass $\geq 200 \mathrm{ml}$ \\
\hline Mediastinal mass & $\begin{array}{l}\text { Tumor diameter }>1 / 3 \text { of the maximal thoracic } \\
\text { diameter of an upright PA chest radiograph }\end{array}$ & ・ Tumor volume $\geq 200 \mathrm{ml}$ \\
\hline
\end{tabular}

PET 5-POINT SCALE (DEAUVILLE CRITERIA) ${ }^{\mathrm{b}}$

\begin{tabular}{|c|l|}
\hline Score & PET/CT scan result \\
\hline 1 & No uptake \\
\hline 2 & Uptake $\leq$ mediastinum \\
\hline 3 & Uptake > mediastinum but $\leq$ liver \\
\hline 4 & Uptake moderately higher than liver \\
\hline 5 & Uptake markedly higher than liver and/or new lesions \\
\hline X & New areas of uptake unlikely to be related to lymphoma \\
\hline
\end{tabular}

With kind permission from Springer International Publishing: Barrington SF, Mikhaeel NG, Kostakoglu L,

et al. Role of imaging in the staging and response assessment of lymphoma: consensus of the Internationa

Conference on Malignant Lymphomas Imaging Working Group. J Clin Oncol 2014;32(27):3048-3058

a Clinical interpretation of staging at diagnosis should not be based on reports alone. Treating clinician notes should summarize interpretation of sites of involvement prior to initiation of treatment.

b PET should be read by an experienced nuclear diagnostic radiologist experienced in reading Deauville scores for PET-adapted therapy. This is a visual analysis and does not include standardized uptake value (SUV).

${ }^{c}$ Volume $=(\mathrm{a} \times \mathrm{b} \times \mathrm{c}) / 2$ where $\mathrm{a}, \mathrm{b}, \mathrm{c}$ are three dimensions of a node or conglomerate

In a later COG study, AHOD0831, the investigators aimed to limit alkylator exposure and decrease radiation volumes in pediatric patients with high-risk HL defined as stage IIIB and IVB $(\mathrm{n}=165$; aged $\leq 21$ years $) .^{42}$ All patients received 2 cycles of ABVE-PC; if they experienced RER, they received an additional 2 cycles of ABVE-PC and IFRT (21 Gy) only to sites of initial bulk. Patients with SER received 2 cycles of ifosfamide and vinorelbine followed by 2 more cycles of ABVE-PC and RT to sites of initial bulk disease and slow-responding sites. According to intent-to-treat analysis, the 4-year second EFS (ie, freedom from second relapse or malignancy) was $91.9 \%$ (95\% CI, 86.1\%-95.3\%). ${ }^{42}$ The 5-year first EFS and OS rates are $79.1 \%$ (95\% CI, 71.5\%-84.8\%) and 95\% (95\% CI, $88.8 \%-97.8 \%$ ), respectively. Although the projected target for second EFS was not reached (ie, 95\%), the EFS and OS rates were comparable to other trials of high-risk $\mathrm{HL}^{41,43}$ In the phase III COG AHOD1331 trial, further refinements are being investigated, including substituting bleomycin with brentuximab vedotin, a CD30 antibodydrug conjugate, the results of which are pending. ${ }^{44,45}$

In the Children's Cancer Group-59704 study, the efficacy of upfront dose intensification with BEACOPP (bleomycin, etoposide, doxorubicin, cyclophosphamide, procarbazine, and prednisone) has been evaluated in pediatric patients with high-risk HL $(\mathrm{n}=99$; age $<21$ years) ${ }^{43,45}$ All patients received 4 cycles of BEACOPP, and patients with rapid response received either 4 cycles of COPP/ABV and no IFRT (female patients) or 2 cycles of ABVD followed by IFRT (21 Gy) (male patients). ${ }^{43}$ Patients who were slow responders received an additional 4 cycles of BEACOPP and IFRT. The 5-year EFS and OS rates were $94 \%$ and $97 \%$, respectively. ${ }^{43}$ Although this regimen is effective at maintaining disease control, it is likely to be associated with increased long-term toxicities. ${ }^{45}$

\section{NCCN Recommendations for High-Risk CHL}

For patients with stage IIB, IIIA, IIIB, and IV CHL, the panel recommends enrollment in an ongoing clinical trial or treatment according to AHOD1331 (based on AHOD0831) or EuroNet-PHL-Cl as the preferred strategies (see PHL5, page 738). Note, not all patients with IIB disease have historically been treated as high risk. Refer to staging algorithm for IIB treatment options (see PHL-2, page 735).

For AHOD1331 regimen, after 2 initial cycles of ABVE-PC, patients with adequate response (rapidly 
PRINCIPLES OF SYSTEMIC THERAPY

Treatment for Relapsed or Refractory Disease

- Consider the following when selecting re-induction or subsequent therapy:

- Referral to a center with expertise given lack of data

- Clinical trial enrollment

- Primary therapy and prior RT exposure

- Cumulative short- and long-term toxicity

- Opportunity to harvest stem cells

- Fertility preservation (option for some patients); refer to fertility clinic for further discussion when able prior to initiation of chemotherapy.

- Consider use of RT as part of therapy for relapsed/refractory disease.

- Additional options may be considered for patients over the age of 18, see NCCN Guidelines for Hodgkin Lymphoma.

\begin{tabular}{|c|c|c|c|}
\hline \multicolumn{4}{|c|}{ Relapsed/Refractory Disease } \\
\hline & $\begin{array}{l}\text { Re-Induction Therapy Options } \\
\text { (in alphabetical order) }\end{array}$ & $\begin{array}{l}\text { Subsequent Therapy Options }{ }^{d} \\
\text { (in alphabetical order) }\end{array}$ & |Maintenance (post-transplant) \\
\hline $\mathrm{CHL}$ & $\begin{array}{l}\text { - Brentuximab vedotin + bendamustine } \\
\text { - Brentuximab vedotin + gemcitabinec, } \\
\text { - Brentuximab vedotin + nivolumab } \\
\text { - DHAP (dexamethasone, cytarabine, cisplatin) } \\
\text { - GV (gemcitabine, vinorelbine) } \\
\text { - IEP-ABVD (ifosfamide, etoposide, prednisone; } \\
\text { - doxorubicin, bleomycin, vinblastine, dacarbazine) } \\
\text { - IGEV (ifosfamide, gemcitabine, vinorelbine) } \\
\text { - IV (Ifosfamide, vinorelbine) }{ }^{11}\end{array}$ & 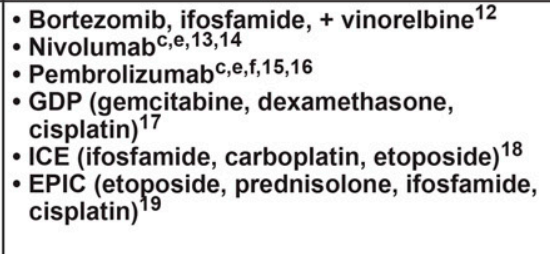 & $\begin{array}{l}\text { Useful in certain circumstances, } \\
\text { for select high-risk } \text { patients: }^{\text {- Brentuximab vedotin }} \text { h,20 }\end{array}$ \\
\hline
\end{tabular}

b Reasonable to try multiple different re-induction regimens as needed prior to ASCR to minimize disease burden with a goal of achieving a metabolic CR prior to transplant.

c Should be considered in patients heavily pretreated (with platinum or anthracycline-based chemotherapy) or if a decrease in cardiac function is observed.

d Subsequent therapy options include re-induction options that were not previously used.

e Emerging data are showing utility as a re-induction option; consider for subsequent therapy if not previously used.

f Pembrolizumab is indicated for the treatment of pediatric patients with refractory CHL, or who have relapsed after 2 or more prior lines of therapy.

9 High-risk: any patient with progressive disease, refractory disease, or relapse within 1 year of original diagnosis.

$\mathrm{h}$ For relapsed CHL, brentuximab vedotin is indicated for the treatment of adult patients after failure of autologous hematopoietic stem cell transplant (HSCT) or after

failure of at least two prior multi-agent chemotherapy regimens in patients who are not auto-HSCT candidates. It is not currently approved for pediatric patients.

responding lesions) are treated with 3 additional cycles of ABVE-PC and ISRT to sites of LMA. Patients with inadequate response (slow responding lesions) receive 3 additional cycles of ABVE-PC and ISRT to sites of LMA. The addition of boost is dependent on PET-positive lesions at end of chemotherapy (see PHL-A 1 of 2, page 741).

For treatment based on EuroNet-PHL-C1, after 2 initial cycles of OEPA, patients with adequate response are treated with 4 cycles of COPDAC. Patients with inadequate response are treated with 4 cycles of COPDAC and ISRT to all sites and boost to sites of inadequate response (see PHL-A 1 of 2, page 741). In both cases, based on an end of therapy PET assessment, patients may either be followed up or considered for biopsy to confirm persistent active disease.

In certain circumstances, BEACOPP and ABVD regimens may be considered for some pediatric patients. ${ }^{35-39,43}$ The panel recommends referring to the adult NCCN Guidelines for Hodgkin Lymphoma (available at NCCN.org) to review relevant data and context. It is worth noting that in the NCCN Guidelines for Hodgkin Lymphoma in adults, regimens with reduced numbers of cycles of BEACOPP have been developed.

\section{Follow-Up After Completion of Treatment}

Given the long-term risks of the therapies for HL, including secondary cancers, cardiac toxicity, pulmonary toxicity, thyroid dysfunction, and reproductive issues, ${ }^{46-54}$ patients should be followed up by an oncologist who is aware of these risks and complications, in coordination with the primary care provider, especially during the first 2 years after treatment (see PHL-6, page 739). The follow-up schedule should be individualized, depending on clinical circumstances such as patient's age, gender, stage of disease, and initial treatment modality.

The panel recommends an interim history and physical examination every 3 to 4 months for 1 to 2 years, then every 6 to 12 months until year 3 , and then annually until 5 years. Recommended laboratory studies include: complete blood count with differential, ESR or C-reactive protein, and a chemistry profile as clinically indicated. If the patient's neck was treated with RT, thyroid stimulating hormone should be evaluated annually. If patients were exposed to regimens that contain bleomycin or pulmonary RT, or have significant pulmonary involvement, or other clinical concerns, PFTs should be considered. At the end of therapy, an echocardiogram may be 


\section{PRINCIPLES OF RADIATION THERAPY}

\section{General Principles}

- Treatment with photons, electrons, or protons may all be appropriate, depending on clinical circumstances.

- In specific instances, advanced RT technologies may be used to spare important organs at risk (OARs) and decrease the risk for late normal tissue damage while still achieving the primary goal of local tumor control.

- Advanced technologies include intensity-modulated RT (IMRT)/volumetric modulated arc therapy (VMAT), breath hold or respiratory gating and/or image-guided RT (IGRT), or proton therapy may offer significant and clinically relevant advantages.

- OARs: heart (including coronary arteries, valves, and left ventricle), lungs, kidneys, spinal cord, esophagus, carotid arteries, bone marrow, breasts, stomach, muscle/soft tissue, and salivary glands.

- Dose-sparing for OARs reflects best clinical practice, as it reduces the risk of late complications from normal tissue damage. Achieving highly conformal dose distributions is especially important for patients who are being treated with curative intent or who have long life expectancies following therapy.

- Breath hold techniques have been shown to decrease incidental dose to the heart and lungs in many disease presentations, including mediastinal HL. Strategies include:

- 4D-CT for simulation or deep inspiration breath hold (DIBH)

- Respiratory gating

- IGRT during treatment delivery

- Since the advantages of these techniques include tightly conformal doses and steep gradients next to normal tissues, target definition and delineation and treatment delivery verification require careful monitoring to avoid the risk of tumor geographic miss and subsequent decrease in tumor control.

- Initial diagnostic imaging with contrast-enhanced CT, MRI, PET, ultrasound, and other imaging modalities facilitate target definition.

- Image guidance may be required to provide assurance of accurate daily delivery.

- Randomized studies to test these concepts are unlikely to be done since these techniques are designed to decrease late effects, which take $10+$ years to develop. In light of that, the modalities and techniques (including proton therapy) that are found to best reduce the doses to the OARs for a given patient in a clinically meaningful way without compromising target coverage should be used.

considered, with repeat echocardiograms thereafter based on specific risk profile (eg, Children's Oncology Group Long Term Follow-up Guidelines).

An annual influenza vaccination and other vaccines per the CDC is recommended for all patients (see the COG Survivorship Guidelines ${ }^{55}$ for more details). In addition, in patients treated with splenic RT, vaccinations should be given before or after RT (ie, pneumococcal, meningococcal, and Haemophilus influenzae type b).

Due to risk of false positives, routine or surveillance PET scans are not recommended. If relapse is suspected (based on imaging, clinical, or pathologic correlations) a PET scan may be recommended. It is acceptable to obtain a CT scan with contrast or MRI of original sites of disease at 3 to 6 -month intervals for up to 2 years after completion of therapy if there are any concerns. Although an MRI scan may substitute CT scan for neck, abdomen, and pelvic regions, a diagnostic CT of the chest is required for imaging of the chest. If the previous PET was positive (Deauville 3-5), a PET/CT or PET/MRI scan is recommended to confirm $\mathrm{CR}$ at the end of all prescribed therapy including RT. The panel notes that once negative, a repeat PET should not be done unless evaluating suspicious findings on the history and physical examination, CT, or MRI. In addition, to minimize false-positive results, it is important to wait at least 8 to 12 weeks after the end of RT to perform PET assessments.

In general, and in terms of monitoring late effects ( $\geq 2$ years after completion of systemic therapy), patients should be encouraged to undergo counseling on issues regarding survivorship, long-term treatment effects (eg, secondary cancers, cardiac disease, and issues affecting the thyroid, bone, and reproductive health), health habits, and psychosocial issues. For comprehensive details, see the COG Survivorship Guidelines. ${ }^{55}$

\section{Relapsed or Refractory CHL}

Although the outcomes for pediatric HL are excellent, approximately $10 \%$ of patients with early-stage disease and up to $25 \%$ of patients with advanced-stage disease experience relapse. ${ }^{8,56,57}$ For patients with relapsed or refractory disease, treatment options include standarddose chemotherapy (reinduction therapy), high-dose chemotherapy with ASCR, or novel approaches. ${ }^{56}$ Reinduction regimens (see PHL-E 2 of 3, page 747) can be divided into 4 major categories. ${ }^{56}$ 


\section{PRINCIPLES OF RADIATION THERAPY}

Volume

- Involved-site RT (ISRT) is recommended as the appropriate field for HL. If the protocol used involved-field RT (IFRT) then it should be replaced by ISRT.

- Planning for ISRT requires CT-based simulation and treatment planning capabilities. Incorporating other modern imaging such as PET and MRI often enhances treatment volume determination.

- ISRT targets the site of the originally involved lymph node(s). The volume encompasses the original or suspected extent of disease prior to chemotherapy or surgery. However, it spares adjacent uninvolved organs (eg, lungs, bone, muscle, kidney) when lymphadenopathy regresses following chemotherapy.

- Pre-chemo or pre-biopsy GTV provides the basis for determining the clinical target volume (CTV). Concerns for questionable subclinical disease and uncertainties in original imaging accuracy or localization may lead to expansion of the CTV and are determined individually using clinical judgment.

- Movement of the CTV by respiration as determined by 4D-CT or fluoroscopy should be used to create an internal target volume (ITV).

- The planning target volume (PTV) is an additional expansion of the CTV that accounts only for setup variations and may differ by site and immobilization technique. Daily image guidance is recommended to minimize the PTV expansion.

- Outline OARs for optimizing treatment plan decisions.

- These should include contouring of breast tissue (conventional breast tissue and glandular breast tissue) and cardiac substructures (left ventricle and coronary vessels), especially when contemporary RT techniques are being used (IMRT, VMAT, and proton therapy).

- The treatment plan can be designed using conventional, 3D conformal RT (3D-CRT), IMRT, or proton therapy techniques using clinical treatment planning considerations of coverage and normal tissue avoidance.

- The treatment of extranodal disease is individualized, but similar principles of GTV/CTV/PTV definition should be applied as for nodal disease.

- Chest wall extension: Effort should be made to include regions of initial chest wall extension to definitive doses.

- Lung involvement:

- Areas of extension into the lung from mediastinal or hilar disease may be treated with lower doses (15 Gy) unless the relative volume is small, in which case higher doses may be utilized.

- Careful consideration of partial lung tolerance is essential.

- Pulmonary nodular disease is usually not treated following chemotherapy unless residual disease is present.

- Pleural or pericardial effusions are not included in the GTV. Nodular pericardial involvement may be included with consideration of cardiac tolerance.

- Bone: Areas of osseous disease may be treated with a CTV expansion beyond the GTV defined by imaging. In the presence of vertebral body disease, the entire vertebra is generally treated.

- If spleen is irradiated, vaccines should be given prior to or after RT (ie, pneumococcal, haemophilus influenzae type b, meningococcal).

1) Platinum-based regimens including: $\mathrm{DHAP}^{58}$ (dexamethasone, cytarabine, and cisplatin); EPIC $^{59}$ (etoposide, prednisolone, ifosfamide, and cisplatin); and, GDP ${ }^{60}$ (gemcitabine, dexamethasone, and cisplatin). In a study of patients with relapsed/refractory $(\mathrm{R} / \mathrm{R})$ HL $(\mathrm{n}=102$; median age, 34 years; range, 21-64 years), the response rate after 2 cycles of DHAP was $89 \%{ }^{58}$ In a retrospective study of 80 children with relapsed or primary refractory $\mathrm{HL}$, treatment with the EPIC regimen $(55 \%$ of patients received stem cell transplantation after first relapse following EPIC regimen) resulted in a 5-year OS and progression-free survival from relapse of $75.8 \%$ and $59.9 \%$, respectively. ${ }^{59}$ In a study of patients with R/R HL ( $n=23$; median age, 36 years; range, 19-57 years) the response rate after 2 cycles of GDP was $69.5 \% .^{60}$

2) Ifosfamide-/etoposide-based regimens including: ICE $^{61}$ (ifosfamide, carboplatin, and etoposide); IEP-ABVD $^{62}$ (ifosfamide, etoposide, prednisone, doxorubicin, bleomycin, vinblastine, and dacarbazine); $\mathrm{IV}^{63}$ (ifosfamide, and vinorelbine); and a regimen composed of bortezomib and IV. ${ }^{64}$ The ICE regimen was developed to decrease nonhematologic toxicities observed with cisplatin containing regimens. ${ }^{57,61}$ In a study of patients with primary refractory or relapsed HL $(n=65$; median age, 27 years; range, 12-59 years), after treatment with 2 biweekly cycles of ICE, patients who responded to therapy received HDT/ASCR and IFRT. In this study, the response rate to ICE was $88 \%$ and the EFS for patients who underwent transplantation was $68 \%{ }^{61}$

In a study of patients with progressive or relapsed HL $(\mathrm{n}=167$; median age, 14.7 years; range, $4.3-24.5$ years), patients were treated with 2 to 3 cycles of IEP alternating with 1 to 2 cycles of ABVD, and supplemented by additional chemotherapy. ${ }^{62}$ Involved disease sites were also treated with individualized doses of RT. After 10 years, the disease-free survival (DFS) and OS rates were $62 \%$ and $75 \%$, respectively. ${ }^{62}$ This study also identified 3 risk groups, defined as progressive disease on or within 3 months of primary treatment, which had the worst prognosis (DFS/ OS rates of $41 \%$ and $51 \%$, respectively); early relapse 3-12 


\section{PRINCIPLES OF RADIATION THERAPY}

- In general, RT fields and doses should be delivered per protocol guidelines used for systemic therapy.

RT Fields

- ISRT can safely replace IFRT or modified IFRT.

- Residual-site RT should be used only when dictated by the protocol or as a "boost" following standard ISRT.

- Protocols calling for IFRT/ISRT to all sites of disease involvement for stage III/IV disease should be avoided, in favor of a regimen that only irradiates sites that are bulky, or inadequate response (SER/SRL).

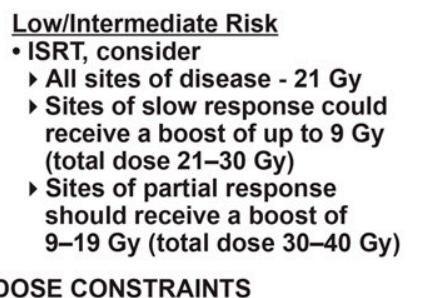

High Risk

- Avoid regimens that require ISRT to all

sites of disease.

-ISRT, consider:

- Bulky disease - 21 Gy

- Slow responding sites could receive a

boost of up to $9 \mathrm{~Gy}$ (total dose 21-30 Gy)

- Partial responding sites should receive a

boost of 9-19 Gy (total dose 30-40 Gy)

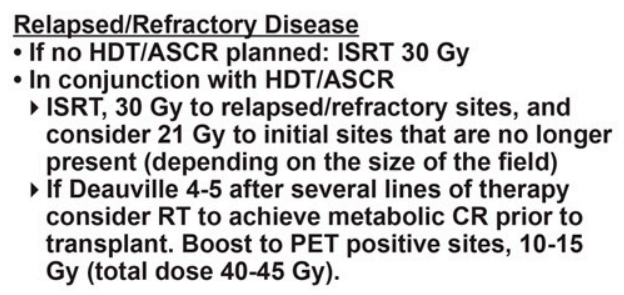

Relapsed/Refractory Disease

- In conjunction with HDT/ASCR

ISRT, 30 Gy to relapsed/refractory sites, and consider $21 \mathrm{~Gy}$ to initial sites that are no longer present (depending on the size of the field) Deauville 4-5 after several lines of therapy transplant. Boost to PET positive sites, 10-15 Gy (total dose 40-45 Gy).

\begin{tabular}{|c|c|c|c|c|}
\hline & Goal* $^{*}$ & Minor Dev & Major Dev & Outcome \\
\hline Mean Heart & ALARA & 10-20 Gy & $>20$ Gy & $\begin{array}{l}\text { Cumulative incidence of significant heart disease at } 30 \text { years: } 10-20 \\
\text { Gy-5.8\%, >20 Gy-7.7\% }{ }^{1}\end{array}$ \\
\hline $\begin{array}{l}\text { Left Ventricle } \\
\text { (mean) }\end{array}$ & $<10$ Gy & 10-15 Gy & $>20$ Gy & $\begin{array}{l}\text { Adult HL: Relative risk of congestive heart failure compared with no } \\
\text { RT: } 16-20 \text { Gy (RR, 1.65), } 21-25 \text { Gy (RR, } 3.84)^{2}\end{array}$ \\
\hline $\begin{array}{l}\text { Lung } \\
\text { (mean) }\end{array}$ & ALARA & 10-15 Gy & $>15 \mathrm{~Gy}$ & $\begin{array}{l}\text { Adult HL: Pulmonary death mean lung } 10-15 \text { Gy (RR, 4.4); 15-20 } \\
\text { Gy (RR, 7.7); Lung fibrosis mean lung dose } 10-15 \mathrm{~Gy}(\mathrm{RR}, 3.8)^{3}\end{array}$ \\
\hline Thyroid & ALARA & V $30<63 \%$ & & Hypothyroidism $11.5 \%$ if $\mathrm{V} 30<62.5 \%$, and $70.8 \%$ if $>62.5 \%{ }^{4}$ \\
\hline $\begin{array}{l}\text { Salivary Glands } \\
\text { (mean) }\end{array}$ & ALARA & & $>24$ Gy & Dose threshold for xerostomia in head and neck cancer ${ }^{5}$ \\
\hline Breast & $\begin{array}{l}\text { ALARA (minimize volume } \\
\text { receiving } 4 \text { Gy or more) }\end{array}$ & & & $\begin{array}{l}\text { Adult HL: } 3.2 \text { fold increase in risk of breast cancer if } 4 \text { Gy delivered } \\
\text { to breast, risk increases with dose }\end{array}$ \\
\hline
\end{tabular}

${ }^{\star} \mathrm{ALARA}=$ as low as reasonably achievable

months from primary treatment with improved OS (DFS/ OS rates of $55 \%$ and $78 \%$, respectively); and late relapse more than 12 months from primary treatment. This had significantly better DFS (DFS/OS rates of $86 \%$ and $90 \%$, respectively), even though this group did not receive stem cell transplantation in second $\mathrm{CR}^{56,62}$

In a study evaluating the efficacy of the IV regimen, 66 patients younger than 30 years of age with R/R HL were treated with 2 cycles of IV. ${ }^{63}$ The overall response rate (ORR) of $72 \%$ allowed most of the patients to undergo subsequent stem cell transplantation. ${ }^{63}$ It is worth noting that this regimen eliminates etoposide, a chemotherapeutic agent associated with secondary malignancy after transplantation. ${ }^{63,65}$ Addition of bortezomib to the IV regimen does not improve anatomic CR after 2 cycles, but may improve the ORR at the completion of therapy ${ }^{64}$

3) Gemcitabine-based regimens including $\mathrm{GV}^{66}$ (gemcitabine and vinorelbine); and IGEV ${ }^{67}$ (ifosfamide, gemcitabine, and vinorelbine). The GV regimen was evaluated in heavily pretreated pediatric patients with R/R HL $(n=30$; median age, 17.7 years; range, 10.7-29.4 years). ${ }^{66}$ All patients had received at least 2 prior chemotherapy regimens and 17 patients had undergone prior autologous stem cell transplantation. Overall, 19 of 25 patients had measurable responses for an observed response rate of $76 \% .{ }^{66}$ Patients who had received transplant before GV tended to respond to therapy over patients who had not received transplant. ${ }^{66}$ In a study of 12 pediatric patients with primary refractory or relapsed HL (age range, 8-16 years), the ORR to IGEV was $100 \%$, with $58 \%$ CRs and $42 \%$ PRs. $^{67}$ The 5-year second EFS and OS rates were $83.0 \%$ $\pm 11.0 \%$ and $90.0 \% \pm 9.5 \%$, respectively. ${ }^{67}$

4) Targeted therapy and immunotherapy-based regimens include brentuximab vedotin combined with bendamustine, ${ }^{68}$ gemcitabine, ${ }^{69}$ or nivolumab $^{70}$; or single-agent nivolumab ${ }^{71,72}$ or single-agent pembrolizumab. ${ }^{73,74}$ In a group of heavily pretreated patients at least 18 years of age with $R / R$ HL $(n=64)$ and anaplastic large cell lymphoma $(\mathrm{n}=1)$, the safety and clinical activity of brentuximab vedotin and bendamustine was evaluated. ${ }^{68}$ An overall response was 
achieved in 29 of 37 patients $(78 \%) .{ }^{68}$ In a COG study (AHOD1221), the safety and efficacy of brentuximab vedotin and gemcitabine was evaluated in children and young adults with primary refractory or early relapsed HL $(n=46$; aged $<30$ years) ${ }^{69}$ Of 42 evaluable patients, 24 (57\%) had a CR within the first 4 cycles of treatment; 4 of 13 patients (31\%) with a PR or stable disease had all target lesions with Deauville scores of $\leq 2$ after 4 cycles of treatment. ${ }^{69}$ Using a Deauville score threshold of $\leq 3,28$ of 42 (67\%) experienced a CR.

The combination of brentuximab vedotin and nivolumab, a human monoclonal PD-1-directed antibody, has been evaluated as initial salvage therapy in adult patients with R/R HL prior to autologous stem cell transplant (ASCT) with a CR rate of $61 \%$ after 4 cycles and no increase in toxicities compared with either agent alone. ${ }^{75}$ For patients who underwent ASCT after the combination, the 2-year progression-free survival was $91 \%{ }^{76}$ In a phase II study of children and AYA patients with R/R HL ( $n=44$; median age range, 9-30 years), patients were treated with 4 cycles of brentuximab vedotin and nivolumab experienced complete metabolic response and ORR of 59\% and $82 \%$, respectively (according to blinded independent central review). ${ }^{70}$ No grade 3 or 4 immune mediated adverse events were observed.

Multiple studies have also demonstrated efficacy of nivolumab and pembrolizumab, another human monoclonal PD-1-directed antibody, in adult patients with R/R HL. ${ }^{71-73}$ In a study evaluating the efficacy of pembrolizumab in pediatric patients with R/R PD-L1-positive solid tumors or lymphomas $(n=154$ evaluable patients; median age, 13 years; interquartile range, 8-15 years), 9 of 15 patients with $\mathrm{R} / \mathrm{R}$ HL experienced an objective response $(60 \%) .{ }^{74}$ In the phase III KEYNOTE-204 study, heavily pretreated adult patients with R/R CHL were randomized to receive either pembrolizumab or brentuximab vedotin $(n=300$ evaluable patients; pembrolizumab arm, $n=148$; brentuximab vedotin arm, $n=152$; aged $\geq 18$ years). ${ }^{77}$ The median progression-free survival in the pembrolizumab treatment arm was statistically longer than the brentuximab vedotin treatment arm (13.2 vs 8.3 months, respectively; hazard ratio, $0.65 ; 95 \% \mathrm{CI}, 0.48-0.88 ; P=.00271) .{ }^{77}$

Because no randomized trials have been conducted to compare reinduction regimens, none of the regimens are considered to be superior to the other. ${ }^{57}$ At this stage, desired qualities in a regimen are low toxicity and high efficacy, and other goals of therapy are to obtain cytoreduction/CR before transplant, and to harvest peripheral blood stem cells for ASCT. ${ }^{56}$

In general, 2 posttransplant treatment options may be considered including: 1) maintenance therapy with brentuximab vedotin (especially useful in patients with high-risk features including progressive disease, refractory disease, or relapse within 1 year of diagnosis) ${ }^{78}$; and 2) RT consolidation after HDT/ASCR depending on previous receipt of RT. Multiple studies support the addition of RT in the transplant setting by showing benefit for local tumor control and improved EFS/OS/DFS..$^{57,79,80}$

\section{NCCN Recommendations for Relapsed/Refractory CHL}

Histologic confirmation with biopsy is recommended before initiating treatment of relapsed/refractory disease (see PHL-7, page 740). If the biopsy is negative, the panel recommends either observation with shortinterval follow-up or additional workup if high index of suspicion for relapse remains. If the biopsy is positive, the panel recommends enrolling the patient in a clinical trial if available, and referral to or consulting with a center of expertise as several options exist for the treatment of relapsed/refractory disease, and lack of data to support one regimen over another.

Typically, patients are treated with reinduction therapies, and after a PET/CT or PET/MRI assessment, if metabolic CR is observed (Deauville score $\leq 3$ ), treatment can be followed up with HDT/ASCR with or without ISRT and with or without maintenance therapy. In general, RT is performed as consolidation after transplant. If unable to achieve a metabolic CR, RT may be used before transplant.

In certain cases, patients may avoid ASCR. These include patients with initial stages other than IIIB or IVB, patients who have no prior exposure to RT, patients with duration of first CR $>1$ year, and patients with no extranodal disease or $\mathrm{B}$ symptoms at relapse. In these patients, reinduction therapy plus ISRT may be considered for initial treatment of R/R HL.

After initial reinduction therapy, an assessment with $\mathrm{PET} / \mathrm{CT}$ or PET/MRI is recommended to evaluate response. If PET-negative, patients may be observed with short-interval follow-up. If PET-positive, subsequent therapy options should be considered, including reinduction options that were not previously used.

\section{Summary}

Pediatric HL is now curable in most patients because of the introduction of more effective and less toxic regimens. However, survivors may experience late treatment-related side effects. For this reason, long-term follow-up is essential after completion of treatment. In addition, improvements in harmonization of staging and response criteria, and risk stratification will improve the therapeutic index. ${ }^{8}$ Emerging data will continue to inform the panel's recommendations and consistent with NCCN philosophy, participation in clinical trials is always encouraged. 


\section{References}

1. Allen CE, Kelly KM, Bollard CM. Pediatric lymphomas and histiocytic disorders of childhood. Pediatr Clin North Am 2015;62:139-165.

2. Jarrett RF. Risk factors for Hodgkin's lymphoma by EBV status and significance of detection of EBV genomes in serum of patients with EBVassociated Hodgkin's lymphoma. Leuk Lymphoma 2003;44(Suppl 3): S27-32.

3. Siegel RL, Miller KD, Jemal A. Cancer statistics, 2020. CA Cancer J Clin 2020;70:7-30.

4. Swerdlow SH, Campo E, Harris NL, et al. WHO Classification of Tumours of Haematopoietic and Lymphoid Tissues, Revised 4th ed. Lyon, France: IARC Press; 2017

5. Siegel RL, Miller KD, Fuchs HE, et al. Cancer Statistics, 2021. CA Cancer J Clin 2021;71:7-33.

6. Ward E, DeSantis C, Robbins A, et al. Childhood and adolescent cancer statistics, 2014. CA Cancer J Clin 2014;64:83-103.

7. Miller KD, Fidler-Benaoudia M, Keegan TH, et al. Cancer statistics for adolescents and young adults, 2020. CA Cancer J Clin 2020;70:443-459.

8. Kelly KM. Hodgkin lymphoma in children and adolescents: improving the therapeutic index. Hematology (Am Soc Hematol Educ Program) 2015; 2015:514-521.

9. Mauz-Körholz C, Metzger ML, Kelly KM, et al. Pediatric Hodgkin Ilymphoma. J Clin Oncol 2015;33:2975-2985.

10. Cheson BD, Fisher RI, Barrington SF, et al. Recommendations for initial evaluation, staging, and response assessment of Hodgkin and nonHodgkin lymphoma: the Lugano classification. J Clin Oncol 2014;32: 3059-3068.

11. El-Galaly TC, d'Amore F, Mylam KJ, et al. Routine bone marrow biopsy has little or no therapeutic consequence for positron emission tomography/computed tomography-staged treatment-naive patients with Hodgkin lymphoma. J Clin Oncol 2012;30:4508-4514.

12. Salaun PY, Gastinne T, Bodet-Milin C, et al. Analysis of 18F-FDG PET diffuse bone marrow uptake and splenic uptake in staging of Hodgkin's lymphoma: a reflection of disease infiltration or just inflammation? Eur J Nucl Med Mol Imaging 2009;36:1813-1821.

13. Purz S, Mauz-Körholz C, Körholz D, et al. [18F]Fluorodeoxyglucose positron emission tomography for detection of bone marrow involvement in children and adolescents with Hodgkin's lymphoma. J Clin Oncol 2011; 29:3523-3528

14. Carbone PP, Kaplan HS, Musshoff K, et al. Report of the Committee on Hodgkin's Disease Staging Classification. Cancer Res 1971;31:18601861.

15. Lister TA, Crowther D, Sutcliffe SB, et al. Report of a committee convened to discuss the evaluation and staging of patients with Hodgkin's disease: Cotswolds meeting. J Clin Oncol 1989;7:1630-1636.

16. Flerlage JE, Kelly KM, Beishuizen A, et al. Staging Evaluation and Response Criteria Harmonization (SEARCH) for Childhood, Adolescent and Young Adult Hodgkin Lymphoma (CAYAHL): Methodology statement [published online January 18, 2017]. Pediatr Blood Cancer, doi: 10.1002 pbc. 26421

17. Kluge R, Kurch L, Georgi T, et al. Current role of FDG-PET in pediatric Hodgkin's lymphoma. Semin Nucl Med 2017;47:242-257.

18. McCarten KM, Nadel HR, Shulkin BL, et al. Imaging for diagnosis, staging and response assessment of Hodgkin lymphoma and non-Hodgkin lymphoma. Pediatr Radiol 2019;49:1545-1564.

19. Voss SD, Cairo MS. Surveillance imaging in pediatric lymphoma. Pediatr Radiol 2019;49:1565-1573.

20. Barrington SF, Mikhaeel NG, Kostakoglu L, et al. Role of imaging in the staging and response assessment of lymphoma: consensus of the International Conference on Malignant Lymphomas Imaging Working Group. J Clin Oncol 2014;32:3048-3058.

21. Meignan M, Gallamini A, Haioun C, et al. Report on the Second International Workshop on interim positron emission tomography in lymphoma held in Menton, France, 8-9 April 2010. Leuk Lymphoma 2010;51: 2171-2180.

22. Meignan M, Gallamini A, Itti E, et al. Report on the Third International Workshop on Interim Positron Emission Tomography in Lymphoma held in Menton, France, 26-27 September 2011 and Menton 2011 consensus. Leuk Lymphoma 2012;53:1876-1881.

23. Barrington SF, Qian W, Somer EJ, et al. Concordance between four European centres of PET reporting criteria designed for use in multicentre trials in Hodgkin lymphoma. Eur J Nucl Med Mol Imaging 2010; 37:1824-1833.
24. Barrington SF, Kluge R. FDG PET for therapy monitoring in Hodgkin and non-Hodgkin lymphomas. Eur J Nucl Med Mol Imaging 2017; 44: (Suppl 1):97-110.

25. Schaefer NG, Taverna C, Strobel K, et al. Hodgkin disease: diagnostic value of FDG PET/CT after first-line therapy-is biopsy of FDG-avid lesions still needed? Radiology 2007;244:257-262

26. Filippi AR, Ragona R, Piva C, et al. Optimized volumetric modulated arc therapy versus 3D-CRT for early stage mediastinal Hodgkin lymphoma without axillary involvement: a comparison of second cancers and heart disease risk. Int J Radiat Oncol Biol Phys 2015;92:161-168.

27. Hoppe BS, Flampouri S, Su Z, et al. Effective dose reduction to cardiac structures using protons compared with 3DCRT and IMRT in mediastinal Hodgkin lymphoma. Int J Radiat Oncol Biol Phys 2012;84:449-455.

28. Hoppe BS, Flampouri S, Zaiden R, et al. Involved-node proton therapy in combined modality therapy for Hodgkin lymphoma: results of a phase 2 study. Int J Radiat Oncol Biol Phys 2014;89:1053-1059.

29. Hoskin PJ, Díez P, Williams M, et al. Recommendations for the use of radiotherapy in nodal lymphoma. Clin Oncol (R Coll Radiol) 2013;25:4958.

30. Giulino-Roth L, Keller FG, Hodgson DC, et al. Current approaches in the management of low risk Hodgkin lymphoma in children and adolescents. Br J Haematol 2015;169:647-660.

31. Dörffel W, Lüders $H$, Rühl $U$, et al. Preliminary results of the multicenter trial GPOH-HD 95 for the treatment of Hodgkin's disease in children and adolescents: analysis and outlook. Klin Padiatr 2003;215:139-145.

32. Mauz-Körholz C, Hasenclever D, Dörffel W, et al. Procarbazine-free OEPA-COPDAC chemotherapy in boys and standard OPPA-COPP in girls have comparable effectiveness in pediatric Hodgkin's lymphoma: the GPOH-HD-2002 study. J Clin Oncol 2010;28:3680-3686.

33. Landman-Parker J, Wallace H, Hasenclever D, et al. First International Inter-Group Study for Classical Hodgkin Lymphoma in Children and Adolescents: EuroNet-PHL-C1 European protocol Euronet PHL-C1; Report of the latest interim Analysis [Abstract\# P064]. Haematologica; 10th International Symposium on Hodgkin Lymphoma Symposium (ISHL10) 2016;101:35

34. Keller FG, Castellino SM, Chen L, et al. Results of the AHOD0431 trial of response adapted therapy and a salvage strategy for limited stage, classical Hodgkin lymphoma: a report from the Children's Oncology Group. Cancer 2018;124:3210-3219.

35. Bhethanabhotla S, Jain S, Kapoor G, et al. Outcome of pediatric advanced Hodgkin lymphoma treated with ABVD and predictors of inferior survival: a multicenter study of 186 patients. Leuk Lymphoma 2017;58: 1617-1623.

36. Jain S, Kapoor G, Bajpai R. ABVD-based therapy for Hodgkin lymphoma in children and adolescents: lessons learnt in a tertiary care oncology center in a developing country. Pediatr Blood Cancer 2016;63:10241030.

37. Marr KC, Connors JM, Savage KJ, et al. ABVD chemotherapy with reduced radiation therapy rates in children, adolescents and young adults with all stages of Hodgkin lymphoma. Ann Oncol 2017;28:849-854.

38. Stieglitz E, Dinh T, Phelps AS, et al. ABVD without radiation for newly diagnosed pediatric and young adult patients with Hodgkin lymphoma: a single center retrospective analysis of 28 consecutive patients. J Pediatr Hematol Oncol 2018;40:290-294.

39. Zubizarreta PA, Alfaro E, Guitter M, et al. Children and adolescent Hodg kin lymphoma in Argentina: long-term results after combined ABVD and restricted radiotherapy. J Pediatr Hematol Oncol 2017;39:602-608.

40. Friedman DL, Chen L, Wolden S, et al. Dose-intensive response-based chemotherapy and radiation therapy for children and adolescents with newly diagnosed intermediate-risk hodgkin lymphoma: a report from the Children's Oncology Group Study AHOD0031. J Clin Oncol 2014;32 3651-3658.

41. Schwartz CL, Constine LS, Villaluna D, et al. A risk-adapted, responsebased approach using ABVE-PC for children and adolescents with intermediate- and high-risk Hodgkin lymphoma: the results of P9425. Blood 2009:114:2051-2059.

42. Kelly KM, Cole PD, Pei Q, et al. Response-adapted therapy for the treatment of children with newly diagnosed high risk Hodgkin lymphoma (AHOD0831): a report from the Children's Oncology Group. Br J Haematol 2019;187:39-48.

43. Kelly KM, Sposto R, Hutchinson R, et al. BEACOPP chemotherapy is a highly effective regimen in children and adolescents with high-risk Hodgkin lymphoma: a report from the Children's Oncology Group. Blood 2011;117:2596-2603. 
44. Castellino SM, Parsons SK, Pei Q, et al. A randomized phase III trial of brentuximab vedotin (Bv) for de novo high-risk classical Hodgkin lymphoma $(\mathrm{CHL})$ in children and adolescents - study design and incorporation of secondary endpoints in Children's Oncology Group (COG) AHOD1331. Accessed May 4, 2021. Available at: https:// childrensoncologygroup.org/ahod1331

45. Kelly KM. Management of children with high-risk Hodgkin lymphoma. Br J Haematol 2012;157:3-13.

46. Amini A, Murphy $B$, Cost $C R$, et al. Cardiac mortality in children and adolescents with Hodgkin's lymphoma: a Surveillance, Epidemiology and End Results Analysis. J Adolesc Young Adult Oncol 2016;5:181-186.

47. Bhakta N, Liu Q, Yeo F, et al. Cumulative burden of cardiovascular morbidity in paediatric, adolescent, and young adult survivors of Hodgkin's lymphoma: an analysis from the St Jude Lifetime Cohort Study. Lancet Oncol 2016;17:1325-1334.

48. Clausen CT, Hasle H, Holmqvist AS, et al. Hyperthyroidism as a late effect in childhood cancer survivors - an Adult Life after Childhood Cancer in Scandinavia (ALiCCS) study. Acta Oncol 2019;58:227-231.

49. Dörffel W, Riepenhausenl M, Lüders H, et al. Secondary malignancies following treatment for Hodgkin's lymphoma in childhood and adolescence. Dtsch Arztebl Int 2015;112:320-327.

50. Fernandez-Pineda I, Davidoff AM, Lu L, et al. Impact of ovarian transposition before pelvic irradiation on ovarian function among long-term survivors of childhood Hodgkin lymphoma: a report from the St. Jude Lifetime Cohort Study. Pediatr Blood Cancer 2018;65:e27232.

51. Inskip PD, Veiga LHS, Brenner AV, et al. Hypothyroidism after radiation therapy for childhood cancer: a report from the Childhood Cancer Survivor Study. Radiat Res 2018;190:117-132.

52. O'Brien MM, Donaldson SS, Balise RR, et al. Second malignant neoplasms in survivors of pediatric Hodgkin's lymphoma treated with lowdose radiation and chemotherapy. J Clin Oncol 2010;28:1232-1239.

53. Oeffinger KC, Hudson MM, Mertens AC, et al. Increasing rates of breast cancer and cardiac surveillance among high-risk survivors of childhood Hodgkin lymphoma following a mailed, one-page survivorship care plan. Pediatr Blood Cancer 2011;56:818-824.

54. van Dalen EC, Caron HN, Dickinson HO, et al. Cardioprotective interventions for cancer patients receiving anthracyclines. Cochrane Database Syst Rev 2011;2011:CD003917.

55. Children's Oncology Group. Long-term follow-up guidelines for survivors of childhood, adolescent, and young adult cancers. 2018. Accessed January 31, 2019. Available at: http://survivorshipguidelines.org/pdf/ 2018/COG_LTFU_Guidelines_v5.pdf

56. Daw S, Wynn R, Wallace H. Management of relapsed and refractory classical Hodgkin lymphoma in children and adolescents. Br J Haematol 2011;152:249-260.

57. Daw S, Hasenclever D, Mascarin M, et al. Risk and response adapted treatment guidelines for managing first relapsed and refractory classical Hodgkin lymphoma in children and young people. Recommendations from the EuroNet Pediatric Hodgkin Lymphoma Group. HemaSphere 2020;4:e329.

58. Josting A, Rudolph C, Reiser M, et al. Time-intensified dexamethasone/ cisplatin/cytarabine: an effective salvage therapy with low toxicity in patients with relapsed and refractory Hodgkin's disease. Ann Oncol 2002;13:1628-1635.

59. Shankar A, Hayward J, Kirkwood A, et al. Treatment outcome in children and adolescents with relapsed Hodgkin lymphoma-results of the UK HD3 relapse treatment strategy. Br J Haematol 2014;165:534-544.

60. Baetz T, Belch A, Couban S, et al. Gemcitabine, dexamethasone and cisplatin is an active and non-toxic chemotherapy regimen in relapsed or refractory Hodgkin's disease: a phase II study by the National Cancer Institute of Canada Clinical Trials Group. Ann Oncol 2003;14:1762-1767.

61. Moskowitz $\mathrm{CH}$, Nimer SD, Zelenetz AD, et al. A 2-step comprehensive high-dose chemoradiotherapy second-line program for relapsed and refractory Hodgkin disease: analysis by intent to treat and development of a prognostic model. Blood 2001;97:616-623.

62. Schellong G, Dörffel W, Claviez A, et al. Salvage therapy of progressive and recurrent Hodgkin's disease: results from a multicenter study of the pediatric DAL/GPOH-HD study group. J Clin Oncol 2005;23:6181-6189.
63. Trippett TM, Schwartz CL, Guillerman RP, et al. Ifosfamide and vinorelbine is an effective reinduction regimen in children with refractory/relapsed Hodgkin lymphoma, AHODOOP1: a children's oncology group report. Pediatr Blood Cancer 2015;62:60-64.

64. Horton TM, Drachtman RA, Chen L, et al. A phase 2 study of bortezomib in combination with ifosfamide/vinorelbine in paediatric patients and young adults with refractory/recurrent Hodgkin lymphoma: a Children's Oncology Group study. Br J Haematol 2015;170:118-122.

65. Krishnan A, Bhatia S, Slovak ML, et al. Predictors of therapy-related leukemia and myelodysplasia following autologous transplantation for lymphoma: an assessment of risk factors. Blood 2000;95:1588-1593.

66. Cole PD, Schwartz CL, Drachtman RA, et al. Phase II study of weekly gemcitabine and vinorelbine for children with recurrent or refractory Hodgkin's disease: a children's oncology group report. J Clin Oncol 2009;27:1456-1461.

67. Marr K, Ronsley R, Nadel H, et al. Ifosfamide, gemcitabine, and vinorelbine is an effective salvage regimen with excellent stem cell mobilization in relapsed or refractory pediatric Hodgkin lymphoma. Pediatr Blood Cancer 2020;67:e28167.

68. O'Connor OA, Lue JK, Sawas A, et al. Brentuximab vedotin plus bendamustine in relapsed or refractory Hodgkin's lymphoma: an international, multicentre, single-arm, phase 1-2 trial. Lancet Oncol 2018;19:257-266.

69. Cole PD, McCarten KM, Pei $\mathrm{Q}$, et al. Brentuximab vedotin with gemcitabine for paediatric and young adult patients with relapsed or refractory Hodgkin's lymphoma (AHOD1221): a Children's Oncology Group, multicentre single-arm, phase 1-2 trial. Lancet Oncol 2018;19:1229-1238.

70. Cole PD, Mauz-Körholz C, Mascarin M, et al. Nivolumab and brentuximab vedotin (BV)-based, response-adapted treatment in children, adolescents, and young adults (CAYA) with standard-risk relapsed/refractory classical Hodgkin lymphoma (R/R cHL): primary analysis. J Clin Oncol 2020;38(Suppl):8013-8013.

71. Armand P, Shipp MA, Ribrag V, et al. Programmed death-1 blockade with pembrolizumab in patients with classical Hodgkin lymphoma after brentuximab vedotin failure. J Clin Oncol 2016;34:3733-3739.

72. Younes A, Santoro A, Shipp M, et al. Nivolumab for classical Hodgkin's lymphoma after failure of both autologous stem-cell transplantation and brentuximab vedotin: a multicentre, multicohort, single-arm phase 2 trial. Lancet Oncol 2016;17:1283-1294.

73. Chen R, Zinzani PL, Fanale MA, et al. Phase II study of the efficacy and safety of pembrolizumab for relapsed/refractory classic Hodgkin lymphoma. J Clin Oncol 2017;35:2125-2132.

74. Geoerger B, Kang HJ, Yalon-Oren M, et al. Pembrolizumab in paediatric patients with advanced melanoma or a PD-L1-positive, advanced, relapsed, or refractory solid tumour or lymphoma (KEYNOTE-051): interim analysis of an open-label, single-arm, phase 1-2 trial. Lancet Oncol 2020;21:121-133.

75. Herrera AF, Moskowitz AJ, Bartlett NL, et al. Interim results of brentuximab vedotin in combination with nivolumab in patients with relapsed or refractory Hodgkin lymphoma. Blood 2018;131:1183-1194.

76. Moskowitz AJ, Herrera AF, Manley T, et al. Brentuximab vedotin and nivolumab for relapsed or refractory classic Hodgkin lymphoma: long-term follow-up results from the single-arm phase 1/2 study. Blood 2019;134 (Suppl 1):238-238.

77. Kuruvilla J, Ramchandren R, Santoro A, et al. KEYNOTE-204: randomized, open-label, phase III study of pembrolizumab (pembro) versus brentuximab vedotin (BV) in relapsed or refractory classic Hodgkin lymphoma (R/R cHL). J Clin Oncol 2020;38(Suppl):8005-8005.

78. Moskowitz $\mathrm{CH}$, Nademanee A, Masszi $\mathrm{T}$, et al. Brentuximab vedotin as consolidation therapy after autologous stem-cell transplantation in patients with Hodgkin's lymphoma at risk of relapse or progression (AETHERA): a randomised, double-blind, placebo-controlled, phase 3 trial. Lancet 2015;385:1853-1862.

79. Kahn S, Flowers $C, X u$ Z, et al. Does the addition of involved field radiotherapy to high-dose chemotherapy and stem cell transplantation improve outcomes for patients with relapsed/refractory Hodgkin lymphoma? Int J Radiat Oncol Biol Phys 2011;81:175-180.

80. Wilke C, Cao Q, Dusenbery KE, et al. Role of consolidative radiation therapy after autologous hematopoietic cell transplantation for the treatment of relapsed or refractory Hodgkin lymphoma. Int J Radiat Oncol Biol Phys 2017;99:94-102 


\section{Individual Disclosures for the NCCN Pediatric Hodgkin Lymphoma Panel}

\begin{tabular}{|c|c|c|c|c|}
\hline Panel Member & $\begin{array}{l}\text { Clinical Research Support/ } \\
\text { Data Safety Monitoring } \\
\text { Board }\end{array}$ & $\begin{array}{l}\text { Scientific Advisory Boards, } \\
\text { Consultant, or Expert } \\
\text { Witness }\end{array}$ & $\begin{array}{l}\text { Promotional Advisory } \\
\text { Boards, Consultant, or } \\
\text { Speakers Bureau }\end{array}$ & Specialties \\
\hline Saro Armenian, DO, MPH & None & None & None & $\begin{array}{l}\text { Pediatric Oncology, and } \\
\text { Hematology/Hematology } \\
\text { Oncology }\end{array}$ \\
\hline Ellen C. Benya, MD & None & None & None & Diagnostic Radiology \\
\hline Adam J. Bobbey, MD & None & None & None & Diagnostic Radiology \\
\hline Stacy Cooper, MD & None & Pfizer Inc. & None & $\begin{array}{l}\text { Hematology/Hematology } \\
\text { Oncology }\end{array}$ \\
\hline Don W. Coulter, MD & None & None & None & Pediatric Oncology \\
\hline Branko Cuglievan, MD & None & None & None & Pediatric Oncology \\
\hline Jamie E. Flerlage, MD, MS & $\begin{array}{l}\text { Seattle Genetics, Inc., } \\
\text { and Teva Pharmaceutical } \\
\text { Industries Ltd. }\end{array}$ & None & None & Pediatric Oncology \\
\hline Bradford S. Hoppe, MD, MPH & None & None & None & $\begin{array}{l}\text { Radiotherapy/Radiation } \\
\text { Oncology }\end{array}$ \\
\hline Leidy Isenalumhe, MD, MS & None & None & None & $\begin{array}{l}\text { Pediatric Oncology, and } \\
\text { Hematology/Hematology } \\
\text { Oncology }\end{array}$ \\
\hline Kara Kelly, MD & $\begin{array}{l}\text { Bristol-Myers Squibb } \\
\text { Company, and Merck \& } \\
\text { Co., Inc. }\end{array}$ & Merck \& Co., Inc. & None & Pediatric Oncology \\
\hline Leslie Kersun, MD, MSCE, MSEd & None & None & None & $\begin{array}{l}\text { Pediatric Oncology, and } \\
\text { Hematology/Hematology } \\
\text { Oncology }\end{array}$ \\
\hline Adam J. Lamble, MD & None & None & None & Pediatric Oncology \\
\hline Martha Pacheco, MD & None & None & None & Pediatric Oncology \\
\hline Anita P. Price, MD & None & None & None & Diagnostic Radiology \\
\hline Kenneth B. Roberts, MD & None & None & None & $\begin{array}{l}\text { Radiotherapy/Radiation } \\
\text { Oncology }\end{array}$ \\
\hline Christine M. Smith, MD & None & None & None & $\begin{array}{l}\text { Pediatric Oncology, and } \\
\text { Hematology/Hematology } \\
\text { Oncology }\end{array}$ \\
\hline Aliyah R. Sohani, MD & None & $\begin{array}{l}\text { Levin Papantonio PA; } \\
\text { Mersana Therapeutics; and } \\
\text { Seeger Salvas \& Devine } \\
\text { LLP }\end{array}$ & None & Pathology \\
\hline Erin M. Trovillion, MD & None & None & None & $\begin{array}{l}\text { Pediatric Oncology, and } \\
\text { Hematology/Hematology } \\
\text { Oncology }\end{array}$ \\
\hline Emily Walling, MD, MPHS & None & None & None & Pediatric Oncology \\
\hline Ana C. Xavier, MD & None & None & None & Pediatric Oncology \\
\hline
\end{tabular}

The NCCN Guidelines Staff have no conflicts to disclose. 